\title{
Wakefulness Is Promoted during Day Time by PDFR Signalling to Dopaminergic Neurons in Drosophila melanogaster
}

\author{
(D) Sheetal Potdar, ${ }^{1}$ and ${ }^{(D)}$ Vasu Sheeba ${ }^{1,2}$
}

DOI:http://dx.doi.org/10.1523/ENEURO.0129-18.2018 ${ }^{1}$ Behavioural Neurogenetics Laboratory, Evolutionary and Organismal Biology Unit, now known as Evolutionary and Integrative
Biology Unit, Jawaharlal Nehru Centre for Advanced Scientific Research, Bangalore 560064, India and ${ }^{2}$ Behavioural Neurogenetics
Laboratory, Neuroscience Unit, Jawaharlal Nehru Centre for Advanced Scientific Research, Bangalore 560064, India

\section{Visual Abstract}
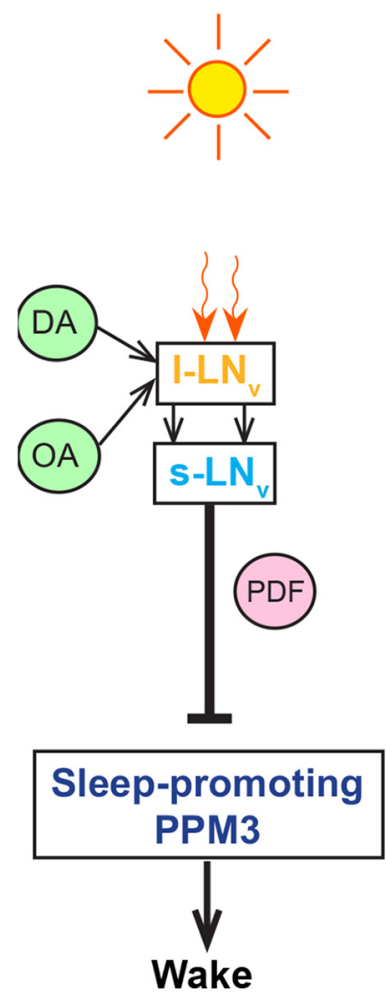
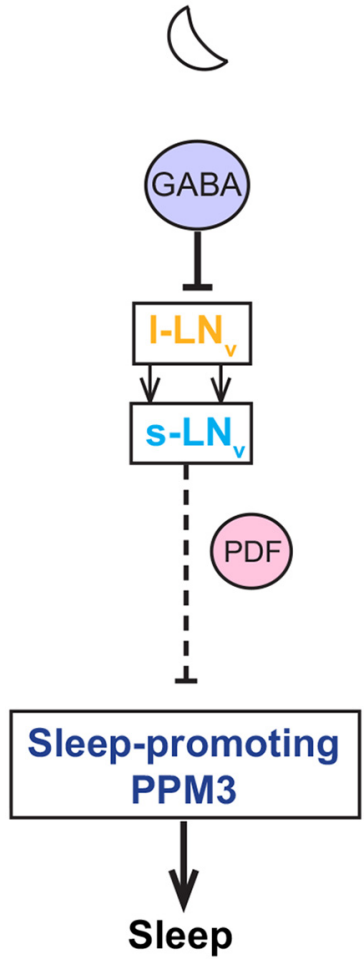

\section{Significance Statement}

It is well established that sleep is controlled by two major factors, the circadian clock as well as a sleep homeostat. Organisms need to remain awake during appropriate times of the day to engage in activities such as feeding and mating that increase their chances of survival. How wakefulness during the day is maintained remains unknown. Here, we show that circadian clock neurons direct their inhibitory peptidergic wake-promoting signal to dopamine neurons specifically during the day time. Importantly, contact of dopamine neurons with known homeostatic structures established through previous studies underlies an important pathway bridging together circadian and homeostatic neurons in sleep/wake regulation. 
Circadian clocks modulate timing of sleep/wake cycles in animals; however, the underlying mechanisms remain poorly understood. In Drosophila melanogaster, large ventral lateral neurons $\left(I-L N_{v}\right)$ are known to promote wakefulness through the action of the neuropeptide pigment dispersing factor (PDF), but the downstream targets of PDF signalling remain elusive. In a screen using downregulation or overexpression (OEX) of the gene encoding PDF receptor (pdfr), we found that a subset of dopaminergic neurons responds to PDF to promote wakefulness during the day. Moreover, we found that $\mathrm{small}_{\mathrm{LN}} \mathrm{LN}_{\mathrm{v}}$ $\left(\mathrm{s}-\mathrm{L} \mathrm{N}_{\mathrm{v}}\right.$ ) and dopaminergic neurons form synaptic contacts, and PDFR signalling inhibited dopaminergic neurons specifically during day time. We propose that these dopaminergic neurons that respond to PDFR signalling are sleep-promoting and that during the day when PDF levels are high, they are inhibited, thereby promoting wakefulness. Thus, we identify a novel circadian clock pathway that mediates wake promotion specifically during day time.

Key words: circadian; dopamine; PDF receptor; sleep; ventral lateral neurons; wake

\section{Introduction}

Daily cycles in several environmental factors synchronize endogenous circadian clocks which drive rhythmic sleep/wake patterns in many organisms. Homeostatic mechanisms modulate the amount and depth of sleep, and also allow animals to recover from any sleep deprivation they may have incurred. Together, these processes control the timing and occurrence of sleep and wake states, thereby modulating sleep/wake cycles. Since the discovery that sleep behavior of Drosophila melanogaster is similar to mammalian sleep in several aspects (Hendricks et al., 2000; Shaw et al., 2000), many pathways and neuronal circuits involving sleep homeostat and circadian clocks have been uncovered. Genes such as minisleep (mns) and hyperkinetic ( $h k$ ) encoding subunits of Shaker potassium channel function in the sleep homeostat (Cirelli et al., 2005; Bushey et al., 2007). More recently, central complex structures such as dorsal fan-shaped body (FB; Donlea et al., 2014) and the ellipsoid body (EB; Liu et al., 2016) have been shown to function as effector and modulator of the sleep homeostat, respectively. Meanwhile, mutations in core circadian clock genes such as Clock $(c / k)$ and Cycle (cyc) have been shown to cause impaired timing of sleep as they tend to become nocturnal (Kumar et al., 2012). The circadian neuropeptide pigment dispersing factor (PDF) and its receptor (PDFR) are involved in

Received April 2, 2018; accepted June 18, 2018; First published July 30, 2018. The authors declare no competing financial interests.

Author contributions: S.P. and V.S. designed research; S.P. performed research; S.P. analyzed data; S.P. and V.S. wrote the paper.

This work was supported by the Science and Engineering Research Board, Department of Science and Technology, India Research Grant SB/SO/AS/019/ 2013 (to V.S.) and intramural funds from the Jawaharlal Nehru Centre for Advanced Scientific Research (to S.P. and V.S.).

Acknowledgements: We thank Fumika Hamada, Gaiti Hasan, Charlotte Helfrich-Forster, Todd C. Holmes, Daniel Kalderon, Gunter Korge, Michael Rosbash, Amita Sehgal, Paul Taghert, and Mark Wu for kindly gifting fly strains; Gaiti Hasan and Todd C. Holmes for generously sharing the anti-TH and anti-PDF (rabbit) antibodies; and Viveka Singh for help with experiments, Sunil Kumar S. for help with imaging, and Rajanna and Muniraju for technical assistance.

Correspondence should be addressed to Vasu Sheeba, Behavioural Neurogenetics Laboratory, Neuroscience Unit, Jawaharlal Nehru Centre for Advanced Scientific Research, Jakkur P.O., Bangalore 560 064, Karnataka, India. E-mail: sheeba@jncasr.ac.in.

DOI:http://dx.doi.org/10.1523/ENEURO.0129-18.2018

Copyright (C) 2018 Potdar and Sheeba

This is an open-access article distributed under the terms of the Creative Commons Attribution 4.0 International license, which permits unrestricted use, distribution and reproduction in any medium provided that the original work is properly attributed. relaying wake-promoting signals from the circadian pacemaker ventral lateral neurons $\left(\mathrm{LN}_{\mathrm{v}} \mathrm{s}\right.$; Parisky et al., 2008; Sheeba et al., 2008; Chung et al., 2009) in response to light input (Shang et al., 2008) as well as dopamine (Shang et al., 2011). While it has been suggested that the EB may be the downstream target of this wake-promoting PDF/ PDFR signaling, the evidence in favor of the same is limited (Parisky et al., 2008).

In the recent past, in the quest to uncover output pathways of the circadian clocks that help in timing of sleep/ wake cycles, a few dedicated circuits have been mapped. Most notably, timing of sleep onset at the beginning of night is a function of increased inhibition of wakepromoting large $L N_{v}\left(I-L N_{v}\right)$ by GABA (Liu et al., 2014). On the other hand, sleep is suppressed at the end of night by the action of PDF on PDFR ${ }^{+}$dorsal neuron $1\left(\mathrm{DN}_{1}\right)$ group of the circadian network which in turn secrete the wakepromoting neuropeptide diuretic hormone 31 (DH31; Kunst et al., 2014). Furthermore, yet another group showed that $\mathrm{DN}_{1} \mathrm{~s}$ through glutamate modulate day-time siesta and night-time sleep by inhibiting the morning (small $L_{\mathrm{v}}$; s- $L N_{v}$ ) and evening (dorsal lateral neurons; $\mathrm{LN}_{\mathrm{d}} \mathrm{s}$ ) activity controlling circadian neurons (Guo et al., 2016). Yet, none of the studies so far have shed light on how circadian neurons may induce wakefulness during the day.

Here, we addressed this question by screening for putative downstream targets of PDFR signaling by altering the levels of pdfr expression in several subsets of neurons - namely, circadian neurons that are known to express pdfr (Hyun et al., 2005; Lear et al., 2005; Mertens et al., 2005; Im and Taghert, 2010) subsets of mushroom body (MB) neurons that are sleep- or wake-promoting (Joiner et al., 2006; Pitman et al., 2006; Cavanaugh et al., 2016), wake-promoting pars intercerebralis (PI; Foltenyi et al., 2007), sleep homeostat EB (Liu et al., 2016), and sleep-promoting FB neurons (Donlea et al., 2011) as well as aminergic neuronal groups, most of which are reported to be wake-promoting (Kume et al., 2005; Crocker et al., 2010). Strikingly, we found that a subset of dopaminergic neurons responds to changes in pdfr expression by changing the levels of day-time sleep, increasing pdfr levels decreases day-time sleep and vice versa. Moreover, we find that $\mathrm{PDF}^{+}$and dopaminergic neurons form synaptic contacts with one another, along with the possibility of the former inhibiting the latter. Thus, our results uncover a dedicated pathway involving signaling from the $\mathrm{PDF}^{+}$neurons perhaps to the PPM3 dopaminergic neurons in the regulation of wakefulness during the day. 
Table 1. Details of fly stocks used in the study.

\begin{tabular}{|c|c|c|}
\hline Fly strains & Source & RRID \\
\hline$w^{1118}$ & BDSC \#5905 & BDSC_5905 \\
\hline$P d f r^{5304}$ & BDSC \#33068 & BDSC_33068 \\
\hline$P d f r^{3369}$ & BDSC \#33069 & BDSC_33069 \\
\hline Pdfr (B) GAL4 & Paul Taghert & BDSC_68215 \\
\hline Cry-39 GAL4 & Todd Holmes & N/A \\
\hline Dvpdf GAL4 & Michael Rosbash & N/A \\
\hline Pdf GAL4 & Todd Holmes & BDSC_6900 \\
\hline Clk 9M GAL4 & Fumika Hamada & BDSC_41810 \\
\hline Clk 4.1M GAL4 & BDSC \#36316 & BDSC_36316 \\
\hline Clk 4.5F GAL4 & BDSC \#37526 & BDSC_37526 \\
\hline OK107 GAL4 & NCBS & $\mathrm{N} / \mathrm{A}$ \\
\hline $201 y$ GAL4 & BDSC \#4440, NCBS & BDSC_4440 \\
\hline c309 GAL4 & BDSC \#6906, NCBS & BDSC_6906 \\
\hline c747 GAL4 & BDSC \#6494, NCBS & BDSC_6494 \\
\hline $30 y$ GAL4 & BDSC \#30818 & BDSC_30818 \\
\hline Dilp2 GAL4 & Amita Sehgal & $\mathrm{N} / \mathrm{A}$ \\
\hline Kurs 45 GAL4 & Gunter Korge & $\mathrm{N} / \mathrm{A}$ \\
\hline Kurs58 GAL4 & Gunter Korge & $\mathrm{N} / \mathrm{A}$ \\
\hline Mai281 GAL4 & Gunter Korge & N/A \\
\hline Маі301 GAL4 & Gunter Korge & $\mathrm{N} / \mathrm{A}$ \\
\hline $121 y$ GAL4 & BDSC \#30815 & BDSC_30815 \\
\hline $104 y$ GAL4 & NCBS & $\mathrm{N} / \mathrm{A}$ \\
\hline c5 GAL4 & BDSC \#30839 & BDSC_30839 \\
\hline c119 GAL4 & BDSC \#30824 & BDSC_30824 \\
\hline c232 GAL4 & BDSC \#30828 & BDSC_30828 \\
\hline Ddc GAL4 & BDSC \#7009 & BDSC_7009 \\
\hline TH GAL4 & BDSC \#8848 & BDSC_8848 \\
\hline Tdc2 GAL4 & BDSC \#9313, NCBS & BDSC_9313 \\
\hline Npf GAL4 & Charlotte Helfrich-Forster & BDSC_25682 \\
\hline UAS Pdfr RNAi & VDRC, KK/110677 & N/A \\
\hline UAS dicer & BDSC \#24651 & BDSC_24651 \\
\hline UAS Pdfr & Paul Taghert & $\mathrm{N} / \mathrm{A}$ \\
\hline$T H-A$ GAL4 & Gaiti Hasan and Mark Wu & $\mathrm{N} / \mathrm{A}$ \\
\hline$T H-C^{\prime} G A L 4$ & Gaiti Hasan and Mark Wu & $\mathrm{N} / \mathrm{A}$ \\
\hline TH-C1 GAL4 & Gaiti Hasan and Mark Wu & N/A \\
\hline TH-D’ GAL4 & Gaiti Hasan and Mark Wu & N/A \\
\hline TH-D1 GAL4 & Gaiti Hasan and Mark Wu & $\mathrm{N} / \mathrm{A}$ \\
\hline TH-D4 GAL4 & Gaiti Hasan and Mark Wu & N/A \\
\hline TH-F2 GAL4 & Gaiti Hasan and Mark Wu & $\mathrm{N} / \mathrm{A}$ \\
\hline TH-F3 GAL4 & Gaiti Hasan and Mark Wu & $\mathrm{N} / \mathrm{A}$ \\
\hline TH-G1 GAL4 & Gaiti Hasan and Mark Wu & $\mathrm{N} / \mathrm{A}$ \\
\hline Pdf LexA & Michael Rosbash & $\mathrm{N} / \mathrm{A}$ \\
\hline LexAop spGFP11/Cyo;UAS spGFP1-10/TM6B & Amita Sehgal & N/A \\
\hline UAS GFP AH2 & BDSC \#6874 & BDSC_6874 \\
\hline Pdfr Myc & Paul Taghert & N/A \\
\hline $\begin{array}{l}\text { LexAOp CD8 GFP-2A-CD8GFP;UAS mLexA VP16 } \\
\text { NFAT,cdc1(H-2,LexAOpCD2GFP/TM6,Tb }\end{array}$ & BDSC \#66542, NCBS & BDSC_66542 \\
\hline UAS NachBac & Todd Holmes & BDSC_9467 \\
\hline UAS dORKNC1 & Todd Holmes & $\mathrm{N} / \mathrm{A}$ \\
\hline UAS Htt QOA & Todd Holmes & $\mathrm{N} / \mathrm{A}$ \\
\hline UAS Htt Q128c & Todd Holmes & $\mathrm{N} / \mathrm{A}$ \\
\hline UAS reaper & Paul Taghert & $\mathrm{N} / \mathrm{A}$ \\
\hline UAS PKAR & Daniel Kalderon & $\mathrm{N} / \mathrm{A}$ \\
\hline UAS PKACA & Daniel Kalderon & N/A \\
\hline UAS ATRPA1 & BDSC \#26263, NCBS & BDSC_26263 \\
\hline
\end{tabular}

BDSC, Bloomington Drosophila Stock Center, Bloomington, Indiana; NCBS, National Center for Biological Sciences, Bangalore, India; VDRC, Vienna Drosophila Resource Center, Vienna, Austria. TH-subset GAL4s that were generated in Mark Wu's lab were obtained from Gaiti Hasan.

\section{Materials and Methods}

Fly strains

Fly strains used along with their source information is listed in Table 1. Briefly, flies were maintained on standard cornmeal medium under LD12:12 at $25^{\circ} \mathrm{C}$. All flies used for sleep measurements have been back-crossed to the standard Iso31 or $w^{1118}$ (BDSC \#5905) background for at least five generations. Pdfr ${ }^{5304}$, Pdfr ${ }^{3369}$, UAS Pdfr RNAi, 
UAS dicer, UAS Pdfr, and TH GAL4 have been backcrossed to $w^{1118}$ for seven generations.

\section{Sleep assays}

Three- to 6-d-old mated females were individually housed in glass tubes (65 $\mathrm{mm}$ in length, $3 \mathrm{~mm}$ in diameter) with sucrose medium (5\% sucrose and $2 \%$ agar) on one end and cotton plug on the other and activity was recorded in DAM2 monitors (Drosophila activity monitoring system, Trikinetics). The DAM system works on the principle that whenever a fly crosses the middle of the tube, it breaks an infra-red beam which gets detected by infrared sensors and recorded as activity counts. Flies were housed in light and temperature-controlled environments with $12 \mathrm{~h}$ of light ( $\sim 300-500$ lux $)$ and $12 \mathrm{~h}$ of darkness (LD12:12) at $25^{\circ} \mathrm{C}$ in incubators (MIR-273, Sanyo; DR36VLC8 Percival Scientific Inc.) for a period of $3 \mathrm{~d}$. Activity was binned at $1 \mathrm{~min}$ and sleep parameters such as daytime and night-time sleep duration, bout length and number and activity per waking minute were estimated using PySolo (Gilestro and Cirelli, 2009), while sleep profiles and sleep latency were obtained from a custom-made Microsoft Excel spreadsheet template.

\section{Statistical analysis}

Change in day-time sleep is calculated as difference between day-time sleep of experimental flies and GAL4 or UAS control flies as a percentage of day-time sleep of GAL4 or UAS control flies. For comparison of sleep parameters, one-way ANOVA with genotype as fixed factor followed by post hoc Tukey's HSD test was conducted. For comparison of GFP fluorescence intensity, two-way ANOVA with genotype and time point as fixed factors followed by post hoc Tukey's HSD test was conducted. Significance level for all tests was set at $p<0.05$.

\section{Immunocytochemistry}

Adult brains were dissected in ice-cold PBS and fixed immediately for 30-40 min in 4\% paraformaldehyde. Fixed brains were blocked in $10 \%$ horse serum for $1 \mathrm{~h}$ at room temperature and $6-9 \mathrm{~h}$ at $4^{\circ} \mathrm{C}$, followed by incubation with cocktail containing primary antibodies for $48 \mathrm{~h}$. The primary antibodies used were anti-GFP (chicken, 1:2000, for GFP labeling and CaLexA measurements, Invitrogen \#A10262, RRID: AB_2534023), anti-PDF (mouse, 1:5000, DSHB, PDF C7, RRID: AB_760350), anti-MYC (mouse, 1:1000, Cell Signaling Technology, \#9B11, RRID: AB_2148465), anti-TH (rabbit, 1:1000, Invitrogen \#P21962, RRID: AB_2539844), anti-GFP (mouse, 1:500, for GRASP, Sigma-Aldrich \#G6539, RRID: AB_259941), and anti-PDF (rabbit, 1:30,000, M. Nitabach and T. C. Holmes). Following seven to eight serial washes with $0.5 \%$ Triton X-100 in PBS (0.5\% PBT), brains were incubated with appropriate secondary antibodies for $24 \mathrm{~h}$. Secondary antibodies were used at a concentration of 1:3000, and they were anti-chicken-Alexa Fluor 488 (Invitrogen, \#A11039, RRID: AB_142924), anti-mouseAlexa Fluor 546 (Invitrogen, \#A11003, RRID: AB_141370), anti-mouse-Alexa Fluor 488 (Invitrogen, \#A11001, RRID: AB_2534069), and anti-rabbit-Alexa Fluor 546 (Invitrogen, \#A11035, RRID: AB_143051). Brains were washed and mounted on glass slides in 7:3 glycerol:PBS medium. Con- focal images were taken on Zeiss LSM 880 (with Airyscan) microscope either with $20 \times, 40 \times$ (oil immersion), or $63 \times$ (oil immersion) objectives.

\section{Results}

\section{PDFR signaling promotes wakefulness specifically during the day}

To establish a phenotype on the basis of which, our screen to uncover downstream targets of PDFR could be designed, we examined two previously established loss-of-function mutants of the pdfr gene, pdfr ${ }^{5304}$ and $p d f r^{369}$. A previous study had reported that both daytime and night-time sleep of these mutants is significantly higher than that of background control flies (Chung et al., 2009). However, we found that both mutants after backcrossing to the widely used Iso31 $\left(w^{1118}\right)$ background for seven to eight generations exhibited significantly higher sleep only during the day-time under a standard LD12:12 cycle at $25^{\circ} \mathrm{C}$ (Fig. $1 A, C ; w^{1118}$ vs $p d f r^{5304}$, Student's two-tailed $t$ test, $t_{(0.05,2,46)}=-2.93, p<0.05 ; w^{1118}$ vs $p d f r^{3369}$, Student's two-tailed $t$ test, $t_{(0.05,2,38)}=-6.33, p<$ $0.00001)$. Moreover, day-time sleep of $p d f r^{5304}$ and pdfr $^{3369}$ mutants was also different from one another (one-way ANOVA, $F_{(2,59)}=21.52, p<0.00001$ ), which was a peculiar observation seen in independent experiments. Functional analysis of the different domains that are deleted in these mutants, the former carries a deletion which excludes all the transmembrane domains and $\mathrm{C}$ terminus while the latter has a smaller deletion with loss of only one transmembrane domain and $\mathrm{C}$ terminus (Hyun et al., 2005) may explain why day-time sleep levels are different in the two mutants. Nonetheless, both mutants sleep much higher as compared to their background controls. Total sleep is significantly higher than the controls only in one of the mutants (Fig. $1 B ; w^{1118}$ vs $p d f r^{5304}$, $t_{(0.05,2,46)}=-1.92, p=0.06 ; w^{1118}{\text { vs } p d f r^{3369}}, t_{(0.05,2,38)}=$ $-3.68, p<0.005)$. However, night-time sleep of both the pdfr mutants was not different from that of the controls (Fig. $1 D ; w^{1118}$ vs $p d f r^{5304}, t_{(0.05,2,46)}=-0.09, p=0.93$; $w^{1118}$ vs $\left.p d f r^{3369}, t_{(0.05,2,38)}=0.13, p=0.9\right)$. These differences in sleep were not due to differences in activity levels (Extended Data Fig. 1-1A,B). Although the activity per waking minute is significantly lower for one of the mutants (Extended Data Fig. $1-1 B ; w^{1118}$ vs pdfr ${ }^{5304}$, $t_{(0.05,2,46)}=2.58, p<0.05 ; w^{1118}$ vs $p d f r^{3369}, t_{(0.05,2,38)}=$ $1.91, p=0.06$ ) as compared to the control, this result was not seen in replicate experiments using the same genotypes (data not shown). Furthermore, the increase in daytime sleep seen in the pdfr mutants is also seen during subjective day time, when these flies are transferred to constant darkness (DD) at $25^{\circ} \mathrm{C}$ (Extended Data Fig. $1-1 C, D ; w^{1118}$ vs $p d f r^{5304}, t_{(0.05,2,41)}=-5.51, p<0.00001$; $w^{1118}$ vs $\left.p d f r^{3369}, t_{(0.05,2,35)}=-5.24, p<0.00001\right)$. Moreover, we confirmed that back-crossing has not resulted in loss of the pdfr mutation by the observation that the behavioural phenotype of advanced evening activity peak (Hyun et al., 2005; Lear et al., 2005; Mertens et al., 2005) is reproduced under LD12:12 (Extended Data Fig. 1-1A). Taken together, these data suggest that absence of func- 


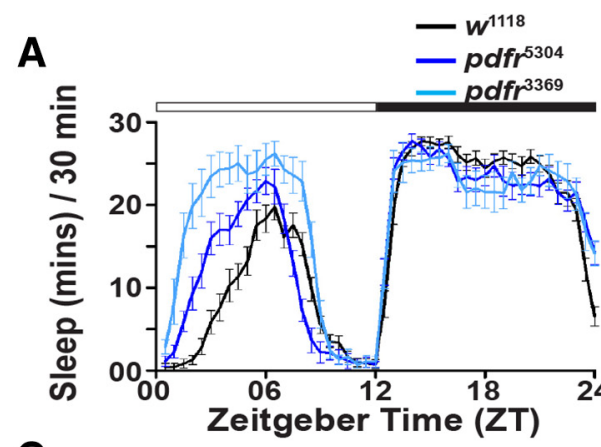

B

C

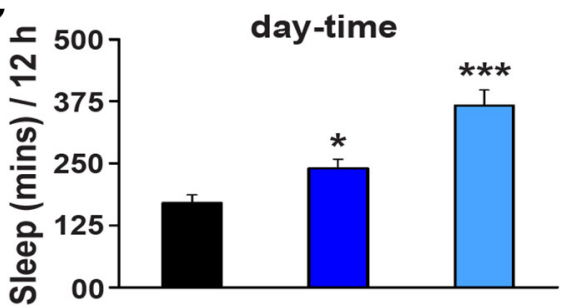

E

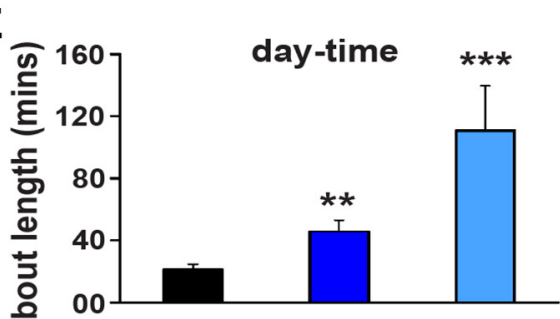

G

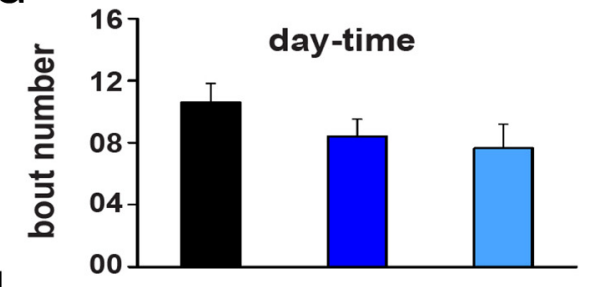

I

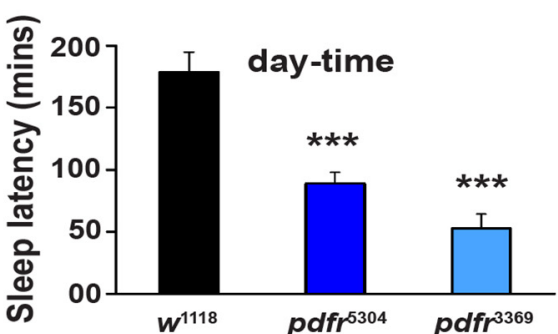

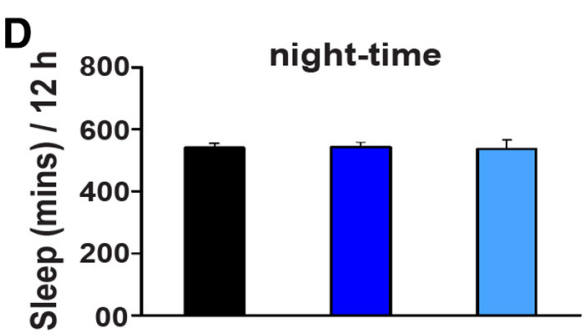

$F$

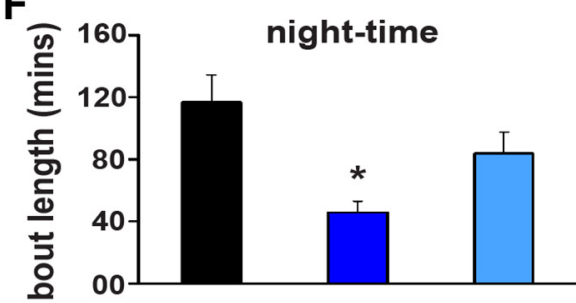

H
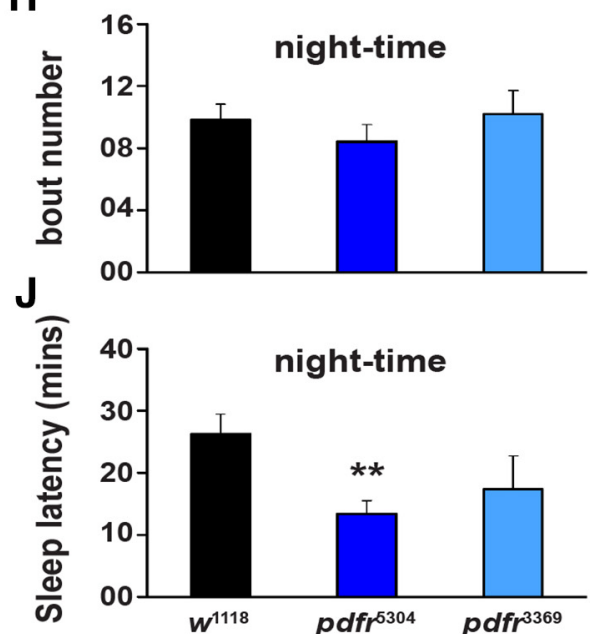

Figure 1. Loss-of-function mutants of $p d f r$ display higher sleep duration during the day. $\boldsymbol{A}$, Amount of time spent sleeping estimated every $30 \mathrm{~min}$ as a function of time-of-day averaged across three cycles. Both pdfr ${ }^{504}$ ( $n=22 \mathrm{flies}$ ) and pdfr ${ }^{3369}$ ( $\left.n=14 \mathrm{flies}\right)$ sleep more during the day time as compared to $w^{1118}\left(n=26\right.$ flies). Night-time sleep of pdfr mutants is similar to that of $w^{1118}$ flies. White and black bars on top indicate $12 \mathrm{~h}$ of day and $12 \mathrm{~h}$ of night, respectively. $\boldsymbol{B}$, Total sleep over the $24-\mathrm{h}$ cycle of $p d f \mathrm{r}^{3369}$ flies is significantly increased as compared to $w^{1118}$ flies, whereas that of $p d f r^{504}$ is not different from $w^{1118}$ flies. $\boldsymbol{C}$, Day-time sleep of both pdfr mutants is significantly higher than that of $w^{1118}$ flies, whereas $(\boldsymbol{D})$ night-time sleep of both pdfr mutants is similar to that of $w^{1118}$ flies. $\boldsymbol{E}$, Average length of sleep bouts during the day is higher in both pdfr mutants as compared to $w^{1118}$, while $(\boldsymbol{F})$ average length of sleep bouts during the night in only pdfr ${ }^{5304}$ mutants is lower than that of $w^{1118}$. Average number of sleep bouts of the pdfr mutants is comparable to that of $w^{1118}$ both during $(\mathbf{G})$ day and $(\boldsymbol{H})$ night. Time taken to fall asleep $(\boldsymbol{I})$ after lights-ON is lower in pdfr ${ }^{5304}$ and $p d f r^{3369}$ mutants as compared to $w^{1118}$ flies and $(\boldsymbol{J})$ after lights-OFF is lower only in pdfr ${ }^{504}$ as compared to $w^{1118}$. Asterisks indicate levels of significance obtained from performing Students' $t$ tests for both mutants comparing each of them to $w^{1118}$ independently; $* p<0.05, * * p<0.005, * * * p<0.0005$. Error bars are SEM. Results representative from two independent experiments. See also Extended Data Figure 1-1. 
tional PDFR results in increased sleep duration specifically during the day.

While the quantity of day-time sleep has increased in the pdfr mutants, the quality of day-time sleep is also different as these pdfr mutants sleep longer within a typical sleep bout during the day time (Fig. $1 E ; w^{1118}$ vs $p d f r^{504}, t_{(0.05,2,46)}=-3.23, p<0.005 ; w^{1118}$ vs $p d f r^{3369}$, $\left.t_{(0.05,2,38)}=-4.17, p<0.0005\right)$. However, the number of such sleep bouts is not different in all three genotypes (Fig. 1G; $w^{1118}$ vs $p d f r^{5304}, t_{(0.05,2,46)}=1.27, p=0.21$; $w^{1118}$ vs $\left.p d f r^{3369}, t_{(0.05,2,38)}=1.43, p=0.16\right)$. These results suggest that sleep is more consolidated during the day time in the absence of functional pdfr. During the night, average sleep bout length is significantly lower in pdfr ${ }^{5304}$ than the control (Fig. 1F; $t_{(0.05,2,46)}=2.15, p<$ $0.05)$, whereas it is comparable to the control in the case of pdfr $^{3369}$ (Fig. $1 F ; t_{(0.05,2,38)}=1.26, p=0.22$ ), and number of sleep bouts during the night of both mutants is similar to that of the control (Fig. $1 H ; w^{1118}$ vs pdff ${ }^{5304}$, $t_{(0.05,2,46)}=-1.24, p=0.22 ; w^{1118}$ vs $p d f r^{3369}, t_{(0.05,2,38)}=$ $-0.2, p=0.84)$. Interestingly, both mutants take lesser amount of time to fall asleep after lights-ON (Fig. 1/; $w^{1118}$ vs $p d f r^{5304}, t_{(0.05,2,46)}=4.16, p<0.00001 ; w^{1118}$ vs $\left.p d f r^{3369}, t_{(0.05,2,38)}=5.3, p<0.00001\right)$, whereas only $p d f^{5304}$ falls asleep sooner than $w^{1118}$ after lights-OFF (Fig. 1J; $w^{1118}$ vs pdfr ${ }^{5304}, t_{(0.05,2,46)}=3.18, p<0.005$; $w^{1118}$ vs $\left.p d f^{3369}, t_{(0.05,2,38)}=0.77, p=0.45\right)$. Given that absence of pdfr leads to increased sleep duration as well as consolidated sleep and makes flies sleep sooner especially during the day time, these results corroborate the previously established role for PDFR signaling mediated by the $\mathrm{PDF}^{+}$neurons in wake-promoting effects (Parisky et al., 2008; Shang et al., 2008; Sheeba et al., 2008; Chung et al., 2009) while highlighting a greater effect on day-time sleep compared to night.

\section{Screen for downstream targets of PDFR signaling}

Previous studies that have characterized the expression pattern of pdfr using different antibodies against PDFR and/or promoter-mediated expression of cellular tags such as myc have revealed pdfr expression in a subset of circadian clock neurons, $\mathrm{PI}, \mathrm{EB}$, and $\sim 50$ as yet non-characterized non-clock cells (Hyun et al., 2005; Lear et al., 2005; Mertens et al., 2005; Parisky et al., 2008; Im and Taghert, 2010). Therefore, on the basis of the predicted expression pattern of $p d f r$ and potential sites in the vicinity of PDF projections, as well as those that function in sleep/wake regulation, we altered expression of $p d f r$ in a total of 26 GAL4 lines including distinct subsets of circadian clock neurons, $\mathrm{MB}, \mathrm{PI}$, central complex, and some neurotransmitter/peptide systems. Our interest was to identify driver lines whose targets responded with both an increase in day-time sleep on downregulation and a decrease in day-time sleep on overexpression (OEX) of pdfr. Moreover, to rule out non-specific effects on daytime sleep of either the GAL4 or UAS parental line, we required the experimental flies to be significantly different as compared to both parental controls to be considered as a hit.
Quite surprisingly, downregulation and/or OEX in subsets of circadian clock neurons, which had previously been reported to modulate activity/rest rhythms in LD as well as in DD (Im and Taghert, 2010) did not show an effect on day-time sleep (Fig. 2). While downregulation of pdfr in 12-14 DN1 ${ }_{\mathrm{p}}$ s using Clk 4.1M GAL4 (Zhang et al., 2010) resulted in a significant increase in day-time sleep as compared to both parental controls (Fig. 2A; Extended Data Fig. 2-1), OEX of pdfr in the same subset of neurons did not result in a corresponding decrease in day-time sleep (Fig. 2B; Extended Data Fig. 2-2). Moreover, downregulation of $p d f r$ in almost all $\mathrm{PDFR}^{+}$clock neurons using Pdfr (B) GAL4 (Im and Taghert, 2010) resulted in an increase in day-time sleep but this was significantly different only from the UAS parental control (Fig. 2A; Extended Data Fig. 2-1). OEX of pdfr using the same driver however resulted in significant decrease in day-time sleep only as compared to the GAL4 control (Fig. 2B; Extended Data Fig. 2-2). Moreover, when pdfr was downregulated and/or overexpressed in a different combination of essentially the same cluster of circadian clock neurons (Cry GAL439; Klarsfeld et al., 2004), consistent effects on day-time sleep were not observed (Fig. 2). These results together lead to the interpretation that circadian clock neurons may not be major downstream targets of PDFR signaling that regulates day-time wakefulness.

In a recent study, it was found that $\mathrm{PDF}^{+}$neurons communicate with DN1s, which then communicate with $\mathrm{DH} 44^{+} \mathrm{PI}$ neurons that brings about rhythmic locomotor activity under DD conditions (Cavanaugh et al., 2014). Given our finding that DN1s are most likely not the downstream targets of $\mathrm{PDF}^{+}$neurons for sleep regulation, we next asked whether the PI neurons were direct recipients of PDF signals for modulation of day-time sleep. Barring a few non-specific parental effects on sleep, none of the 5 PI-specific GAL4 drivers we screened showed any significant effects on day-time sleep when pdfr was downregulated and/or overexpressed (Fig. 2). Thus, although PI neurons appear to be well-placed to receive PDF signals, our finding suggests that they are not required for sleep regulation by the $\mathrm{PDF}^{+}$neurons. In the light of our results and previous findings that PI neurons modulate sleep and wake levels (Foltenyi et al., 2007; Crocker et al., 2010), it appears that the PDF signaling and PI neurons are in different pathways of sleep and wake regulation.

Given that pdfr is expressed in the EB, and the suggestion that they could be the output neurons of PDF effects on sleep and wake levels (Parisky et al., 2008), we downregulated and overexpressed pdfr using GAL4 drivers that distinctly label the EB. We found that downregulation of pdfr using c119 GAL4 led to an increase in day-time sleep (Fig. 2A; Extended Data Fig. 2-1), however it was not accompanied with a corresponding decrease in day-time sleep on OEX of pdfr (Fig. 2B; Extended Data Fig. 2-2). Another GAL4 driver targeting the EB did not show these effects on day-time sleep on downregulation and OEX of pdfr (c232 GAL4; Fig. 2). Thus, these results suggest that EB may not be downstream of PDFR signaling in sleep and wake modulation. 

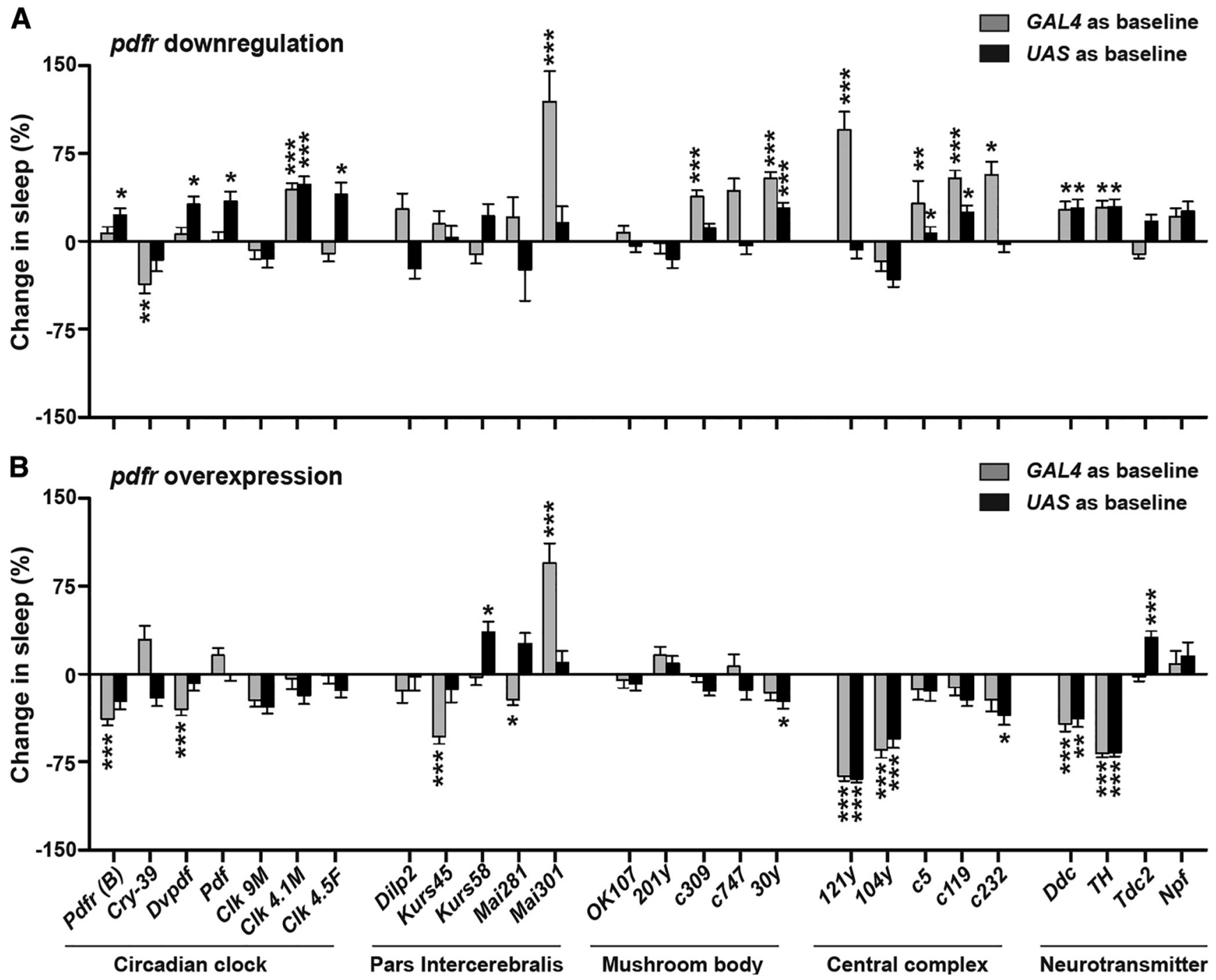

Figure 2. Screen to identify downstream targets of PDFR signaling. A, Downregulation of pdfr using UAS pdfr RNAi; UAS dcr and (B) OEX of pdfr using UAS pdfr crossed with GAL4 lines expressed in subsets of circadian clock neurons, PI, MB, central complex, and major neurotransmitter systems. Bars represent percentage increase (positive values) or decrease (negative values) in day-time sleep of experimental flies with respect to that of either GAL4 (gray) and UAS (black) parental controls. Asterisks above the bars indicate level of significance when a one-way ANOVA with genotype as factor followed by post hoc Tukey's test was done on raw day-time sleep levels. Several lines when used to downregulate pdfr show a significant increase in day-time sleep, but do not show a corresponding decrease in day-time sleep when pdfr is overexpressed (Clk 4.1M GAL4, 30y GAL4, c5 GAL4, c119 GAL4), whereas a few lines show a significant decrease in day-time sleep when pdfr is overexpressed, but no corresponding increase in day-time sleep is seen when pdfr is downregulated (121y GAL4, 104y GAL4). However, in two lines (Ddc GAL4, TH GAL4), when pdfr is downregulated, there occurs a significant increase in day-time sleep, and when pdfr is overexpressed, there occurs a significant decrease in day-time sleep. All other details are as in Figure 1. For all genotypes, $n>24$ flies. Results representative from at least two independent experiments. See also Extended Data Figures 2-1, 2-2.

Im and Taghert (2010) reported that in addition to circadian clock neurons, $\mathrm{PI}$, and $\mathrm{EB}$, there are $\sim 50$ cells in the brain that are PDFR ${ }^{+}$. We hypothesized that these 50 cells could potentially be any one of the MB and/or FB cells, neurons both of which are implicated in sleep regulation (Joiner et al., 2006; Pitman et al., 2006; Donlea et al., 2011; Donlea et al., 2014) and which may lie in the vicinity of projections of the $\mathrm{PDF}^{+} \mathrm{s}-\mathrm{LN}_{\mathrm{v}}$ neurons. Not so surprisingly, none of the GAL4 lines labeling either MB or FB showed significant and opposite effects on day-time sleep on downregulation and OEX of pdfr (Fig. 2). Inter- estingly, however, 4 GAL4 drivers showed strong significant effects on day-time sleep on either downregulation only or OEX only of pdfr. Out of these, when pdfr was downregulated using the 30y GAL4 which labels the $\alpha / \beta$ lobes strongly and the rest of the MB weakly (Aso et al., 2009), day-time sleep was significantly higher as compared to both GAL4 and UAS controls (Fig. 2A; Extended Data Fig. 2-1). Interestingly, OEX of pdfr using broader FB drivers such as $121 y$ GAL4 and 104y GAL4 resulted in decrease of day-time sleep (Fig. 2B; Extended Data Fig. 2-2). However, similar results were not obtained with a 
more restricted driver (c5 GAL4) for FB thereby revealing non-specific effects of the OEX using the broad driver.

We next focused on a few neurotransmitter/peptide clusters that have previously been known to regulate sleep and wake such as dopamine, serotonin, octopamine and neuropeoptide F (NPF; Kume et al., 2005; Yuan et al., 2006; Crocker and Sehgal, 2008; He et al., 2013). To our surprise, when we downregulated pdfr in serotonergic and dopaminergic neurons using Ddc GAL4, as well as dopaminergic neurons alone using TH GAL4, we found that day-time sleep was significantly higher than the parental controls (Fig. 2A; Extended Data Fig. 2-1). Moreover, when we overexpressed pdfr using the same GAL4 drivers, we found that day-time sleep was significantly lesser than the parental controls (Fig. 2B; Extended Data Fig. 2-2). However, there was no significant effect of either downregulating or overexpressing pdfr in either $\mathrm{NPF}^{+}$or octopaminergic neurons on day-time sleep. Taken together, these results suggest that dopaminergic neurons are the most likely candidate for being the downstream targets of PDFR signaling to modulate day-time sleep and wake levels.

\section{PDFR signaling to dopaminergic neurons promotes day-time wakefulness}

We next examined the sleep/wake behavior of flies with downregulated or overexpressed pdfr in dopaminergic neurons in further detail. While downregulation (DR) of $p d f r$ led to increase in day-time sleep and OEX of pdfr in dopaminergic neurons decreased day-time sleep (Fig. $3 A-D$; one-way ANOVA, DR, $F_{(2,89)}=6.53, p<0.005$; OEX, $\left.F_{(2,88)}=43.81, p<0.00001\right)$, interestingly both manipulations of pdfr expression levels led to an increase in night-time sleep (Fig. $3 A, B$; Extended Data Fig. 3-1C,D; $\mathrm{DR}, F_{(2,89)}=12.48, p<0.0005$; OEX, $F_{(2,88)}=43.91, p<$ $0.00001)$. However, these differences in sleep levels were not as a result of changes in activity levels (Extended Data Fig. 3-1A,B; DR, $F_{(2,89)}=2.55, p=0.08$; OEX, $F_{(2,88)}=$ $0.09, p=0.9)$. Not only was the day-time sleep increased when pdfr was downregulated in dopaminergic neurons, the average sleep bout length was significantly longer as compared to both controls (Fig. $3 E ; F_{(2,89)}=16.45, p<$ $0.00001)$, although the number of sleep bouts was not different from the UAS control (Fig. 3G; $F_{(2,89)}=10.33, p$ $<0.0005)$. Interestingly, the flies with downregulated $p d f r$ in dopaminergic neurons took the same amount of time to fall asleep after lights-ON as the controls (Fig. 3/; $F_{(2,89)}=$ $2.59, p=0.08$ ). Flies with overexpressed $p d f r$ in dopaminergic neurons displayed shorter average sleep bouts during the day time (Fig. $\left.3 F ; F_{(2,88)}=13.78, p<0.00001\right)$ as well as lesser number of such sleep bouts (Fig. $3 H$; $\left.F_{(2,88)}=9.45, p<0.0005\right)$. Unlike the pdfr downregulated flies, those with overexpressed $p d f r$ in dopaminergic neurons took longer to fall asleep after lights-ON (Fig 3J; $\left.F_{(2,88)}=28.96, p<0.00001\right)$. Night-time sleep in both manipulations of $p d f$ expression levels was different from the controls only in terms of quantity, not in quality since sleep bout length and number were not affected (Extended Data Fig. 3-1E-J; sleep bout length: DR, $F_{(2,89)}=$ 3.72, $p<0.05$; OEX, $F_{(2,88)}=6.24, p<0.005$; sleep bout number: DR, $F_{(2,89)}=3.98, p<0.05 ;$ OEX, $F_{(2,88)}=4.24$, $p<0.05$. Note that the significant values for $E, G$, and $H$ are due to differences between experimental and only UAS control flies as shown in Extended Data Figure 3-1. Night sleep latency: DR, $F_{(2,89)}=1.1, p=0.34$; OEX, $F_{(2,88)}$ $=2.81, p=0.07)$. Thus, these results lead us to hypothesize that decreasing PDFR signaling to dopaminergic neurons increases day-time sleep, while increasing PDFR signaling to dopaminergic neurons suppresses day-time sleep and makes it fragmented, in addition to delaying sleep onset, suggesting that PDFR signaling to dopaminergic neurons is necessary for initiating and maintaining day-time wakefulness.

\section{PDFR $^{+}$PPM3 neurons modulate day-time wakefulness}

Dopaminergic neurons labeled on the basis of reactivity to antibody against Tyrosine Hydroxylase (anti-TH), which is the rate-limiting enzyme for dopamine synthesis, are divided into several subsets based on their anatomic location (Mao and Davis, 2009). There are two subsets present in the anterior brain (protocerebral anterior medial and lateral; PAM and PAL, respectively) and five subsets in the posterior brain (protocerebral posterior medial and lateral; PPM and PPL, respectively; PPM1-3 and PPL1-2). Of these, two previous studies have implicated a pair of bilaterally located PPL1 neurons (Liu et al., 2012) and a unilateral PPM3 neuron (Ueno et al., 2012) in promoting wakefulness through the inhibition of sleep-promoting FB. We asked whether the PDFR signaling is acting on either or both of these subsets to promote wakefulness specifically during the day. We used the previously created and characterized GAL4 drivers $\left(T H-A, C^{\prime}, C 1, D^{\prime}, D 1, D 4, F 2\right.$, $F 3$, and G1) targeting different subsets of dopaminergic neurons (Liu et al., 2012). When pdfr was downregulated or overexpressed using the TH-A GAL4 which does not drive expression in any of the dopaminergic neurons (Liu et al., 2012), expectedly no difference in the day-time sleep levels was seen (Fig. 4A,B; Extended Data Figs. 4-1, $4-2)$, thus implying no non-specific GAL4 effects. When we specifically targeted the downregulation or OEX to the anterior dopamine subsets PAM and PAL by using the TH-C' and TH-C1 GAL4 drivers, no changes in day-time sleep were observed (Fig. 4A,B; Extended Data Figs. 4-1, 4-2), thus ruling out the involvement of PAM and PAL subsets in receiving PDFR signaling and promoting daytime sleep. The $T H-D, F$ and $G$ drivers are expressed in different subsets of PPM2, PPM3, PPL1 and PPL2 neurons (Extended Data Fig. 4-3; Liu et al., 2012). On applying the same stringent criteria as before, we find that downregulation and OEX of $p d f r$ under the control of $T H-D^{\prime}, D 1$, and $F 3$ drivers result in significant and opposite changes in day-time sleep as compared to both parental controls (Fig. 4A,C; Extended Data Figs. 4-4, 4-1, 4-2). Thus, neurons belonging to PPL1, PPL2, and PPM3 subsets that are common to $T H-D^{\prime}, D 1$, and $F 3$ drivers but not expressed by $T H-D 4, F 2$, and $G 1$ drivers are the likely downstream targets of PDFR signaling important in modulating day-time wakefulness. 
A

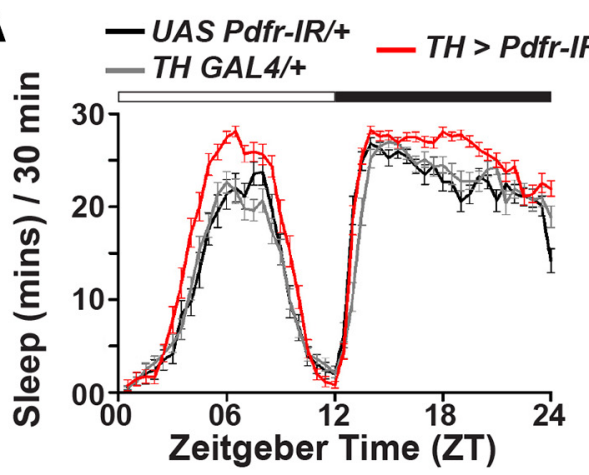

C

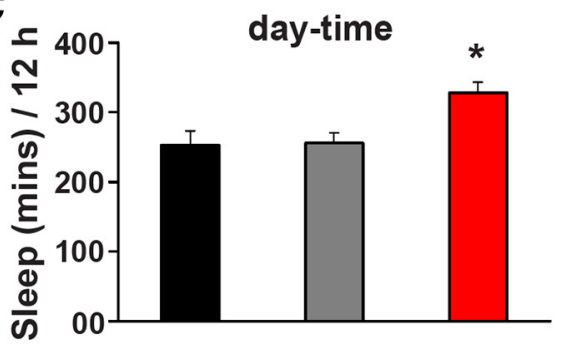

E

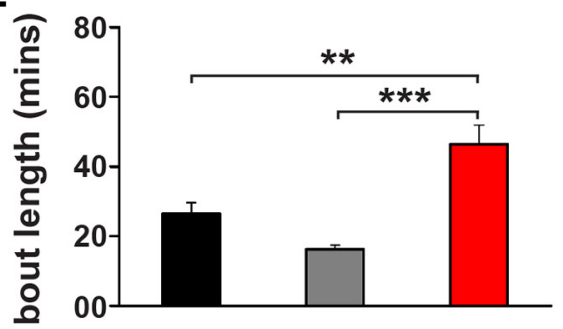

G

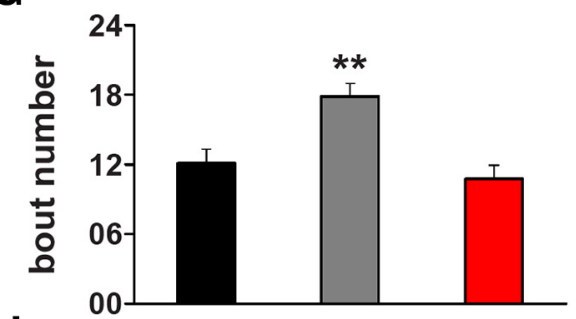

I

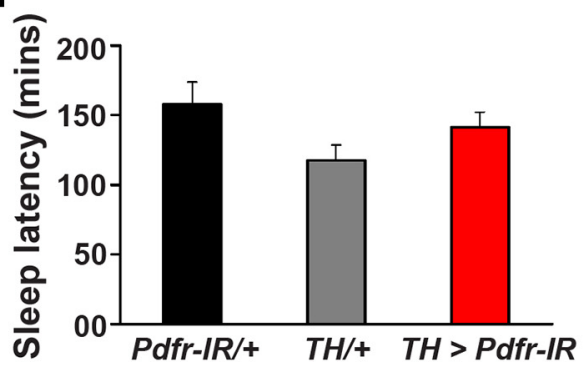

B

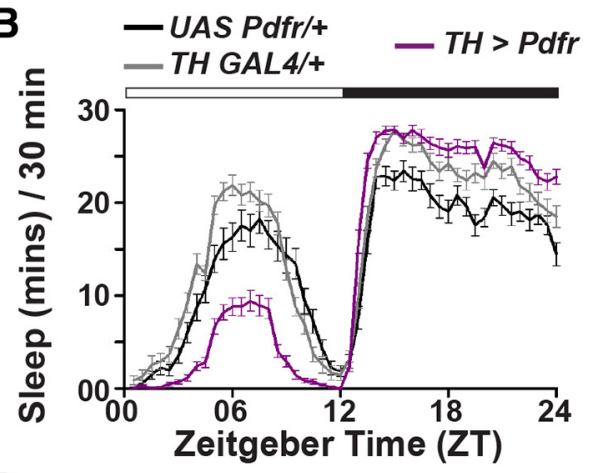

D

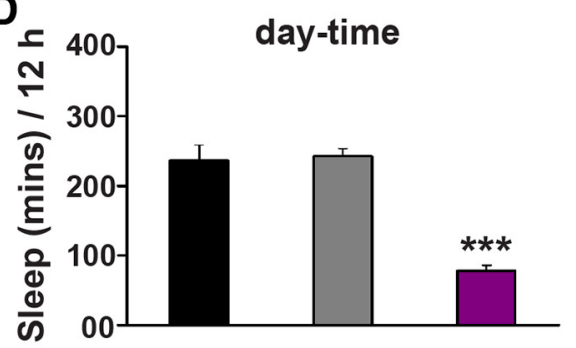

$F$

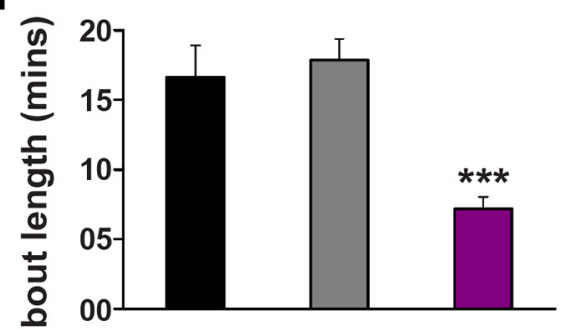

H

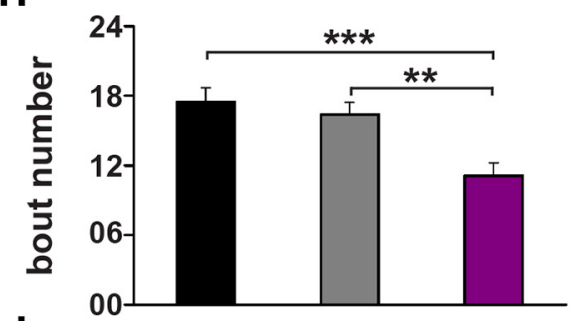

J

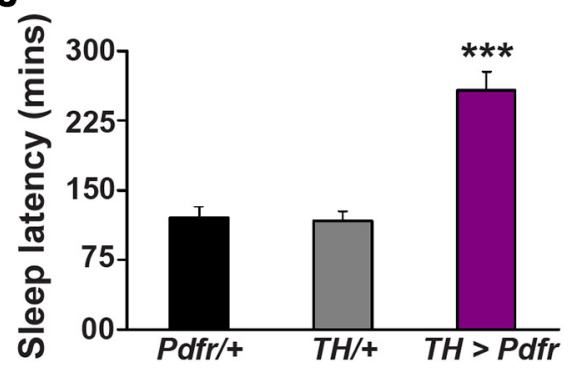

Figure 3. Quantity and quality of day-time sleep changes with changing pdfr expression levels in dopamine neurons. Sleep duration for every 30 min of an average LD12:12 cycle $(\boldsymbol{A})$ when pdfr is downregulated and $(\boldsymbol{B})$ when pdfr is overexpressed in dopaminergic neurons. $\boldsymbol{A}$, Day-time sleep as well as night-time sleep is increased in TH GAL4 > UAS Pdfr RNAi; UAS dcr ( $n=32$ flies) as compared to TH GAL4/+ $(n=31$ flies) and UAS PDFR RNAi/+; UAS dcrl+ ( $n=29$ flies) controls, whereas $(B)$ day-time sleep is decreased but night-time sleep is increased in TH GAL4 $>$ UAS $\operatorname{Pdfr}(n=28$ flies) as compared to TH GAL4/+ $(n=28$ flies $)$ and UAS Pdfr/ $+(n=30$ flies) controls. C, Day-time sleep duration, (E) average sleep bout length, (G) average number of sleep bouts, and (I) latency to fall asleep after lights-ON for TH GAL4> UAS Pdfr RNAi; UAS dcr flies compared to controls. $\boldsymbol{D}$, Day-time sleep duration, $(\boldsymbol{F})$ average sleep bout length, $(\boldsymbol{H})$ average sleep bout number, and $(\boldsymbol{J})$ latency to fall asleep after lights-ON for TH GAL4 > UAS Pdfr flies compared to controls. Asterisks indicate significance levels obtained from one-way ANOVA with genotype as factor followed by post hoc Tukey's test. All other details are as in Figure 1. Results representative from four independent experiments. See also Extended Data Figure 3-1. 
A pdfr downregulation
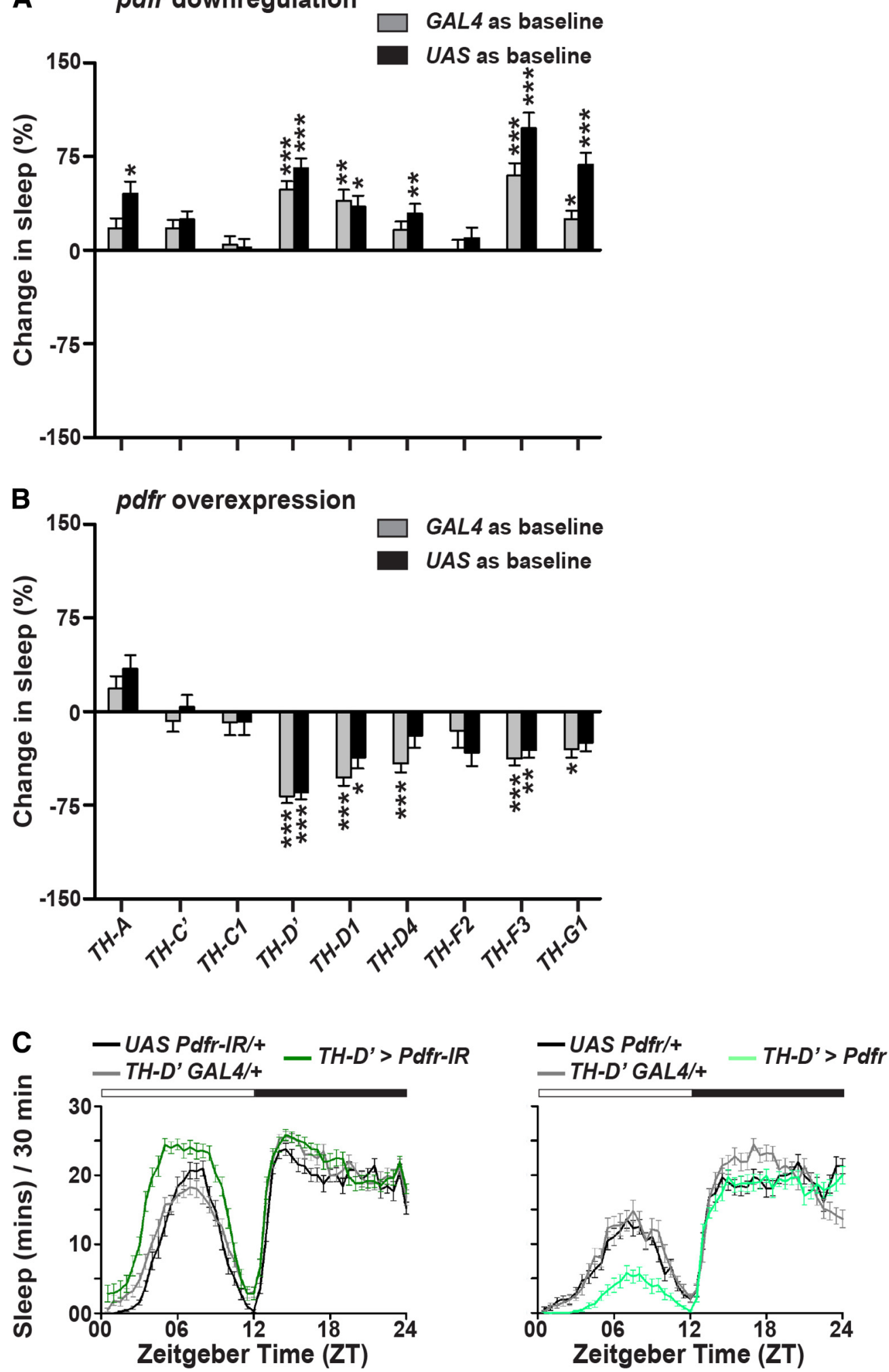

Figure 4. Screen to identify the subset of dopaminergic neurons that are downstream of PDFR signaling. $\boldsymbol{A}$, Downregulation of pdfr using UAS pdfr RNAi; UAS dcr and (B) OEX of pdfr using UAS pdfr crossed with GAL4 lines expressed in different subsets of dopamine neurons. Downregulation and OEX of pdfr using only $T H-D$ ', TH-D1, and TH-F3 GAL4 lines leads to significant and opposite effects on day-time sleep. In all three lines, downregulation of pdfr leads to increase in day-time sleep, whereas OEX of pdfr leads to decrease in day-time sleep. For all genotypes, $n>24$ flies. All other details are as in Figure 2. $\boldsymbol{C}$, Sleep duration for every 30 min of an average LD12:12 cycle of TH-D' GAL4> UAS Pdfr RNAi; UAS dcr ( $n=32$ flies) compared to TH-D' GAL4/+ ( $n=31$ flies) and UAS Pdfr RNAi/+; UAS dcrl+( $n=31$ flies) controls (left) and TH-D' GAL4> UAS Pdfr ( $n=32$ flies) compared to TH-D' GAL4/+ $(n$ 
continued

$=32$ flies) and UAS Pdfr/ $+(n=32$ flies) controls (right). Day-time sleep is increased with downregulation, whereas it is decreased with OEX of pdfr in a subset of dopaminergic neurons driven by TH-D' GAL4. All other details are as in Figure 1. Results representative from at least two independent experiments. See also Extended Data Figures 4-1, 4-2, 4-3, 4-4, 4-5.

To identify the dopaminergic neurons that receive signals from PDF, we used the previously described Pdfr myc line (Im and Taghert, 2010) where myc is fused to the Pdfr gene, such that labeling MYC labels most PDFR ${ }^{+}$ neurons including clock neurons and $\sim 50$ as yet uncharacterized non-clock neurons. We co-stained adult brains of pdfr myc flies with antibodies against TH and MYC and examined any overlap that may exist between $\mathrm{TH}^{+}$and $\mathrm{PDFR}^{+}$neurons. We found that one to two PDFR ${ }^{+}$neurons always lie in the vicinity of PPL1 and PPM3 subset of dopaminergic neurons (Extended Data Fig. 4-5A; $n=22$ hemispheres). On careful examination, we found that in three out of 11 brains visualized, there was one PPM3 neuron in each hemisphere that was both $\mathrm{TH}^{+}$and PDFR $^{+}$(Extended Data Fig. 4-5B, left). The low number of brains showing $\mathrm{TH}^{+}$and $\mathrm{PDFR}^{+}$PPM3 neurons could be because of high background and low affinity of the antiMYC antibody. However, in none of the brains could we detect any overlap between PPL1 $\mathrm{TH}^{+}$and PDFR ${ }^{+}$neurons, although they were quite close to each other (Extended Data Fig. 4-5B, right). Thus, we can only conclude that perhaps one PPM3 neuron per hemisphere may express the PDFR.

\section{$\mathrm{PDF}^{+}$and $\mathrm{TH}^{+}$neurons form synaptic contacts in sLN $\mathrm{N}_{\mathrm{v}}$ axons}

Based on our results with altering pdfr levels that show that dopaminergic neurons are downstream targets of PDFR signaling, we next examined the nature of communication between $\mathrm{PDF}^{+}$and dopaminergic neurons. Because PDF is a neuropeptide, it can have long-range non-synaptic effects on downstream neurons expressing the PDFR (Nässel and Winther, 2010). We conducted a GFP reconstitution across synaptic partners (GRASP) experiment which relies on two independent binary systems allowing the expression of two membrane-bound GFP fragments in different sets of neurons, such that GFP is reconstituted and fluoresces only when the fragments are present at synaptic distances (Gordon and Scott, 2009). A similar experiment done previously had shown the presence of synapses between $\mathrm{PDF}^{+}$and dopaminergic neurons in the $L N_{v}$ dendrites (Shang et al., 2011). However, here, we asked whether synapses between $\mathrm{PDF}^{+}$and dopaminergic neurons occur specifically in the region of $\mathrm{LN}_{\mathrm{v}}$ axons since we wished to examine postsynaptic targets of PDF. We costained adult brains of flies in which dopaminergic neurons expressed GFP1-10 fragment and PDF $^{+}$neurons expressed GFP11 fragment with anti-GFP antibody that specifically labels reconstituted GFP and anti-PDF to visualize the $\mathrm{LN}_{\mathrm{v}}$ projections. We found that reconstituted GFP signal was specifically detected in the ascending part of the dorsal projection of $\operatorname{sLN}_{v}$ (Fig. 5A) which is an axonal process. However, when either fragment was individually driven in the $\mathrm{PDF}^{+}$neurons or dopaminer- gic neurons alone, no GFP signal was detected (Fig. $5 B, C)$ showing that the antibody does not recognize individual fragments of GFP. This shows that $\mathrm{PDF}^{+}$and dopaminergic neurons form synaptic connections especially in the axonal projections of $\mathrm{s}-\mathrm{LN}_{\mathrm{v}}$, thus bolstering our finding that dopaminergic neurons are downstream of PDFR signaling. Furthermore, in brains with dopaminergic neurons labeled with promoter driven GFP (TH GAL4 > UAS GFP), as well as with anti-TH, we find a dopaminergic projection intersecting the ascending dorsal projection of $\mathrm{s}-\mathrm{LN}_{\mathrm{v}}$ (Fig. $5 D$, asterisk).

\section{Auxiliary role of $s L N_{v}$ in mediating wake-promoting effects of I-LN}

While previous studies suggest a negligible role for the $\mathrm{s}-\mathrm{LN}_{\mathrm{v}}$ in the sleep/wake circuit (Shang et al., 2008; Chung et al., 2009), s-LN $N_{v}$ have been proposed to have a secondary role in promoting wake-mediating effects of I-LN (Parisky et al., 2008; Potdar and Sheeba, 2012). To explore their role further, we made use of previously reported toxic version of Huntingtin protein expression $(\mathrm{Htt}$ $Q 128$, referred to as $Q 128$, non-toxic form referred to as Q0) to selectively render $\mathrm{s}-\mathrm{LN}_{\mathrm{v}}$ dysfunctional (Sheeba et al., 2010) while simultaneously changing the electrical properties of the remaining I-LN $\mathrm{v}$ by expressing the bacterial sodium channel NachBac (NB; Nitabach et al., 2006). We compared sleep patterns of the following genotypes of flies: those in which $\mathrm{s}-\mathrm{LN}_{\mathrm{v}}$ were dysfunctional but I- $L N_{v}$ were normally firing $\left(\mathrm{s}^{-} \mathrm{L}^{+} \mathrm{Q} 128\right.$ and NCQ128), some neurons from both $L N_{v}$ subsets were ablated $\left(s^{ \pm} L^{ \pm}\right.$ rpr, apoptotic gene reaper; Potdar and Sheeba, 2012), both $\mathrm{LN}_{v}$ subsets hyperexcited $\left(\mathrm{s}^{\mathrm{H}} \mathrm{L}^{\mathrm{H}} \mathrm{NBQO}\right)$ and $\mathrm{s}-\mathrm{LN} \mathrm{N}_{\mathrm{v}}$ were dysfunctional but I-LN $N_{v}$ were hyperexcited $\left(s^{-} \mathrm{L}^{\mathrm{H}}\right.$ $N B Q 128)$ with their respective controls in which both $L N_{v}$ were normally firing and functional $\left(\mathrm{s}^{+} \mathrm{L}^{+} Q 0\right.$ for $\mathrm{s}^{-} \mathrm{L}^{+}$ Q128, $\mathrm{s}^{+} \mathrm{L}^{+}$GAL4 for $\mathrm{s}^{ \pm} \mathrm{L}^{ \pm}$rpr, $\mathrm{s}^{+} \mathrm{L}^{+} \mathrm{NCQ0}$ for $\mathrm{s}^{\mathrm{H}} \mathrm{L}^{\mathrm{H}}$ $N B Q 0$ ands $^{-} L^{+} N C Q 128, N C$ refers to $d O R K N C 1$, which is a non-conducting potassium channel; Nitabach et al., 2002). Additionally, $\mathrm{s}^{-} \mathrm{L}^{+}$NCQ128 served as control for $\mathrm{s}^{-}$ $\mathrm{L}^{\mathrm{H}}$ NBQ128. Because I-LN $\mathrm{V}_{\mathrm{v}}$ wake-promoting effects are primarily mediated by light (Shang et al., 2008), we examined sleep levels of these flies in LD12:12 cycles with different day-time light intensities. We observe altered levels of day-time sleep only when the $\mathrm{LN}_{\mathrm{v}}$ are ablated, but never when they are hyperexcited either completely or partially (Extended Data Fig. 5-1A, $B$; two-way ANOVA, 10 lux: $F_{(7,478)}=4.5, p<0.0005 ; 300$ lux: $F_{(7,468)}=8.27, p<$ $0.00001 ; 2000$ lux: $\left.F_{(7,476)}=13.91, p<0.00001\right)$. Moreover, the increased levels of day-time sleep in $\mathrm{s}^{ \pm} \mathrm{L}^{ \pm}$flies is seen only when the light intensity is low ( $\sim 10$ lux $)$, but with increasing light intensity, day-time sleep levels are comparable to $\mathrm{s}^{+} \mathrm{L}^{+}$flies, suggesting that remaining $L N_{v}$ that have not been ablated can modulate day-time sleep effectively especially in the presence of saturating light intensities (Extended Data Fig. 5-1A,B, left panels). Fur- 
A Pdf LexA > GFP11; TH GAL4 > GFP1-10
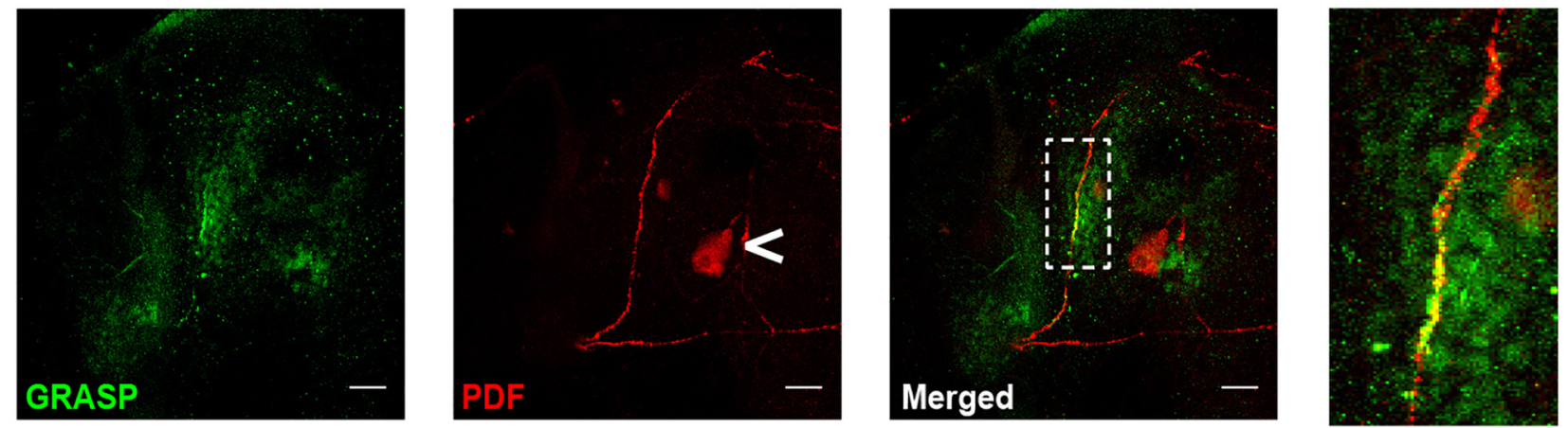

B Pdf LexA > GFP11; GFP1-10
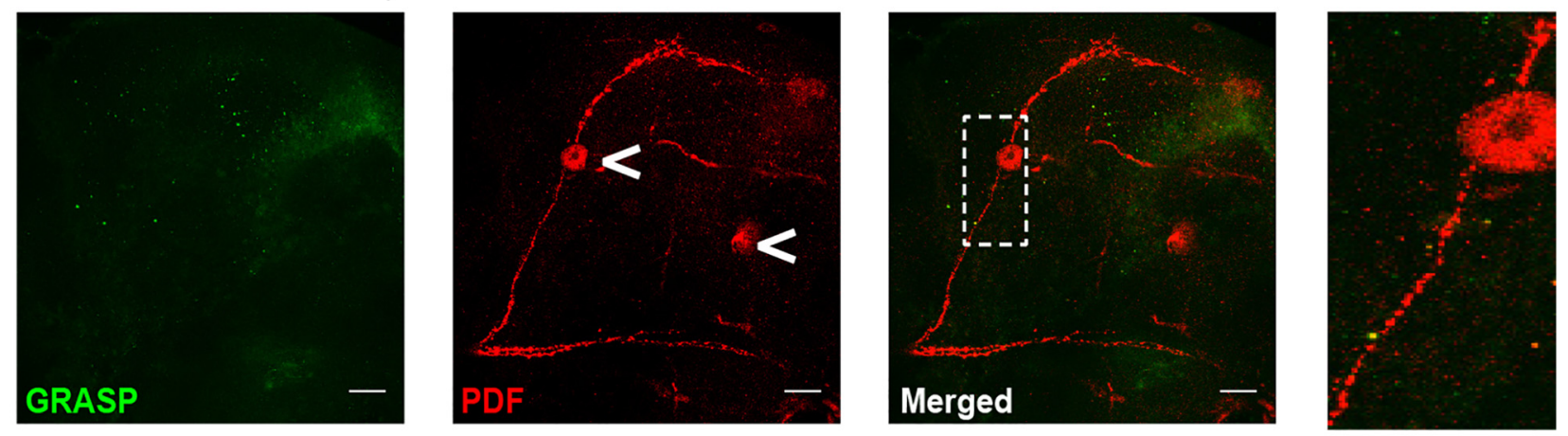

C TH GAL4 > GFP1-10; GFP11
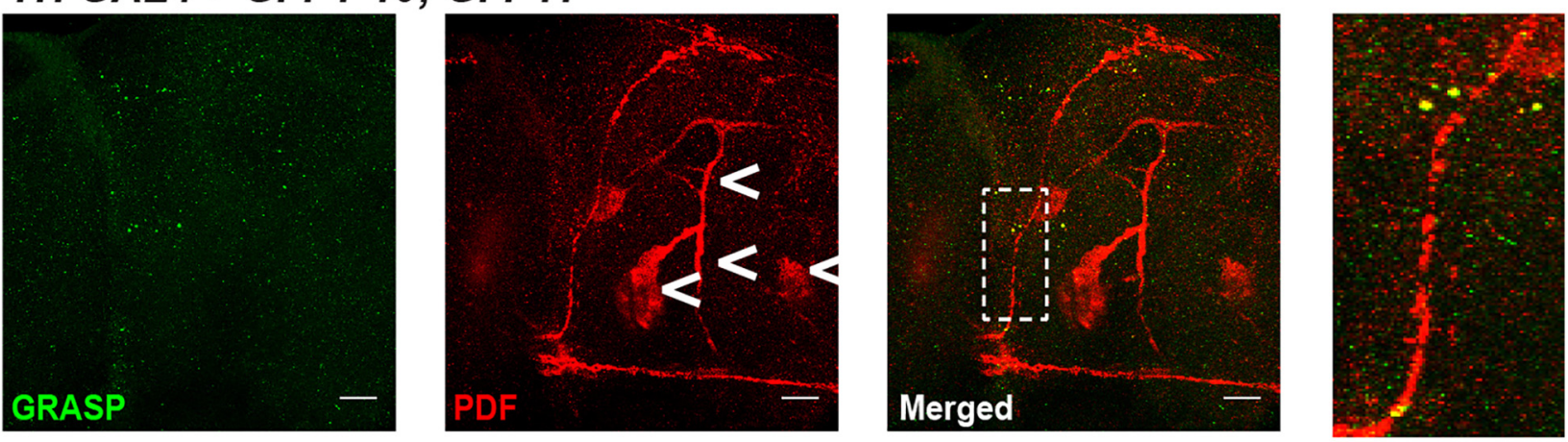

D TH GAL4 > UAS GFP
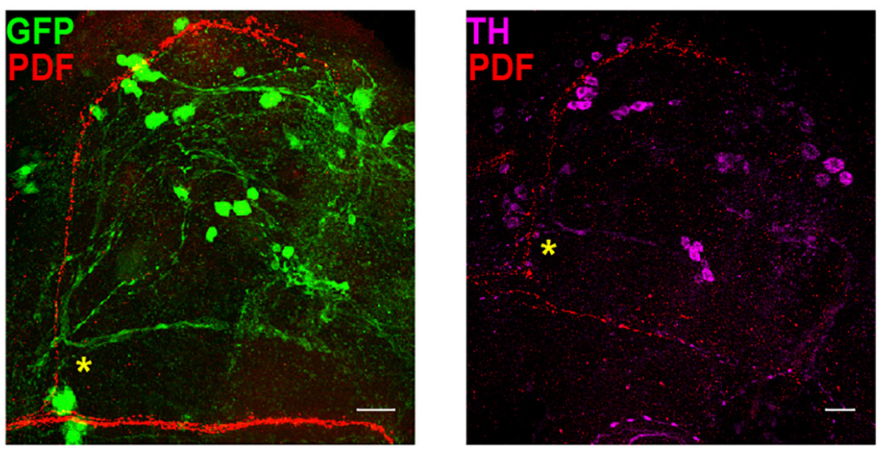

Figure 5. Anatomic connections between $\mathrm{TH}^{+}$and $\mathrm{PDF}^{+}$neurons. $\boldsymbol{A}$, Reconstituted GFP (GRASP) signal was detected in brains of 
continued

flies expressing LexAop CD4::spGFP11 under Pdf LexA control and UAS CD4::spGFP1-10 under TH GAL4 control $(n=22$ hemispheres). GRASP signal colocalized with ascending portion of $s-L N_{v}$ dorsal projections labeled with antibody against PDF. Highlighted region is magnified in the right-most panel. Control flies lacking expression of $(\boldsymbol{B}) \mathrm{spGFP1-10}(n=16 \mathrm{hemispheres)}$ and (C) spGFP11 ( $n=16$ hemispheres) do not show GRASP signal. Results representative from two independent experiments. Arrowheads indicate non-specific staining. Expression of GFP using TH GAL4 and colabeling PDF (D, left) and using antibodies against TH and PDF ( $\boldsymbol{D}$, right) in wild-type flies reveal dopaminergic projection in the vicinity of ascending portion of $s-\mathrm{LN}_{\mathrm{v}}$ dorsal projection as indicated by asterisks. Scale bar: $20 \mu \mathrm{m}$. See also Extended Data Figure 5-1.

thermore, day or night-time sleep levels are not altered when $\mathrm{S}-\mathrm{LN}_{\mathrm{v}}$ are dysfunctional and I-LN $\mathrm{v}$ are normally firing $\left(\mathrm{s}^{-} \mathrm{L}^{+}\right)$. Interestingly, the finding that flies with hyperexcited $\mathrm{LN}_{\mathrm{v}}$ show unchanged day-time sleep levels even in low light intensity LD12:12 cycles suggest that lightresponsive $\mathrm{I}-\mathrm{LN}_{\mathrm{v}}$ can be saturated in terms of their firing capacity with as low light intensity as 10 lux. However, night-time sleep levels are always significantly lower than controls when both $\mathrm{LN}_{\mathrm{v}}$ are hyperexcited $\left(\mathrm{s}^{\mathrm{H}} \mathrm{L}^{\mathrm{H}}\right.$; Extended Data Fig. 5-1A,B, right panels). Interestingly, night-time sleep levels of flies with dysfunctional s-LN $N_{v}$ but hyperexcited I-LN $\mathrm{V}_{\mathrm{v}}\left(\mathrm{s}^{-} \mathrm{L}^{\mathrm{H}}\right)$ is significantly reduced as compared to controls, but always higher than the $\mathrm{s}^{\mathrm{H}} \mathrm{L}^{\mathrm{H}}$ flies (Extended Data Fig. 5-1A, $B$, right panels). In fact, under LD12:12 with low light intensity days (10 lux), we find that night-time sleep levels of $\mathrm{S}^{-} \mathrm{L}^{\mathrm{H}}$ flies are comparable with their $\mathrm{S}^{+} \mathrm{L}^{+}$ controls (Extended Data Fig. 5-1A, $B$, right-top). These results validate that $\mathrm{I}-\mathrm{LN}_{\mathrm{v}}$ modulate wakefulness and further show that functional $\mathrm{s}-\mathrm{LN}_{\mathrm{v}}$ are required to mediate these effects.

\section{PDFR signaling inhibits PPM3 neuronal activity specifically during the day}

Previous studies have shown that binding of PDF to PDFR results in a strong increase of cyclic AMP (cAMP) levels (Mertens et al., 2005; Shafer et al., 2008) and moderate increase of intracellular calcium $\left(\mathrm{Ca}^{2+}\right)$ levels when expressed in HEK293 cells (Mertens et al., 2005). To test whether CAMP is the second messenger involved in mediating wakefulness through PDFR signaling, we overexpressed using TH-F3 GAL4 either the catalytic or regulatory subunit of $\mathrm{cAMP}$-dependent protein kinase $\mathrm{A}$
(PKA; PKACA and PKAR) which increases or reduces PKA activity, respectively. We found no significant changes in day-time sleep as a result of increasing or decreasing PKA activity (Extended Data Fig. 6-1A,B,D; one-way ANOVA, $\left.F_{(2,86)}=0.44, p=0.64\right)$, although daytime sleep of TH-F3 GAL4 > UAS PKAR flies was significantly lower as compared to only the UAS PKAR control flies (Extended Data Fig. 6-1C; one-way ANOVA, $F_{(2,77)}=$ 4.41, $p<0.05)$. This shows that cAMP may not be the secondary messenger responding to PDFR signaling in the $\mathrm{TH}-\mathrm{F}^{+}$neurons, as changing PKA activity levels has negligible effects on day-time sleep.

To assess the functional importance of the connectivity between $\mathrm{PDF}^{+}$and dopaminergic neurons, we next examined intracellular $\mathrm{Ca}^{2+}$ levels in dopaminergic neurons at two time points, one during day [zeitgeber time (ZT)4; 4 $\mathrm{h}$ after lights-ON] and another during night (ZT14) in the presence and absence of functional PDFR (Pdfr ${ }^{5304}$ mutant background). To quantify $\mathrm{Ca}^{2+}$ levels, we used the recently developed CaLexA method which relies on calcium-dependent-nuclear transport of VP16:LexA to drive GFP downstream of LexAop responder element (Masuyama et al., 2012). When the CaLexA transgenes are expressed using the broad dopaminergic driver $\mathrm{TH}$ GAL4, we find that one to two neurons of some dopaminergic subsets notably within the PAL, PPM2, and PPM3 clusters express GFP (Table 2). However, differential expression of GFP depending on time point and genotype is observed only in approximately one to two PPM3 neurons per hemisphere (Table 3). We find that in the presence of functional PDFR, at ZT4 when the levels of PDF are also high (Park et al., 2000), the amount of GFP ${ }^{+}$signal seen in

Table 2. Quantification of GFP positive cell numbers

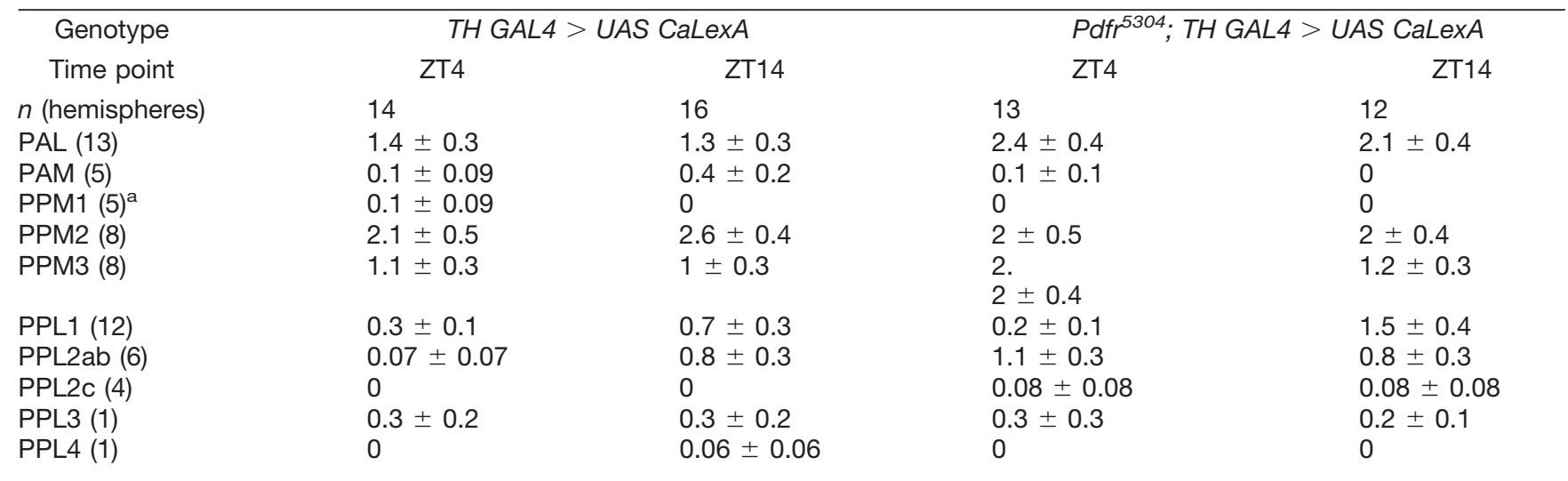

Number of $\mathrm{GFP}^{+}$neurons as seen in different dopaminergic subsets (mean $\pm \mathrm{SEM}$ ) in brain hemispheres expressing CaLexA under the TH GAL4 driver in wild-type and $p d f r^{5304}$ backgrounds during day (ZT4) and night (ZT14) time points. Numbers in parentheses indicate overall number of neurons of different

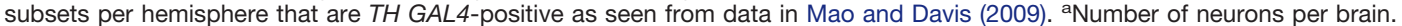


Table 3. Quantification of GFP staining intensity as a proxy for calcium signalling and neuronal activity levels

\begin{tabular}{|c|c|c|c|c|}
\hline \multirow{2}{*}{$\begin{array}{l}\text { Genotype } \\
\text { Time point }\end{array}$} & \multicolumn{2}{|c|}{ TH GAL4 > UAS CaLexA } & \multicolumn{2}{|c|}{ Pdfr ${ }^{5304} ;$ TH GAL4 > UAS CaLexA } \\
\hline & ZT4 & ZT14 & ZT4 & ZT14 \\
\hline n (hemispheres) & 14 & 16 & 13 & 12 \\
\hline PAL & $30.4 \pm 4.8$ & $32.3 \pm 5.7$ & $50.3 \pm 5.9$ & $36.8 \pm 6$ \\
\hline PAM & $27.3 \pm 13.8$ & $55.2 \pm 19.2$ & $36.7 \pm 10.6$ & 0 \\
\hline PPM1 & $6.4 \pm 0.3$ & 0 & 0 & 0 \\
\hline PPM2 & $23.4 \pm 5.2$ & $41.3 \pm 5.9$ & $36.9 \pm 6.7$ & $23.8 \pm 4.3$ \\
\hline PPM3 & $17.2 \pm 3$ & $72.5 \pm 12.1$ & $75.8 \pm 8.7$ & $69.6 \pm 7.6$ \\
\hline PPL2c & 0 & 0 & 18.7 & 33.6 \\
\hline PPL3 & $6 \pm 1.5$ & $23.6 \pm 8.6$ & $11.4 \pm 1.9$ & $52.2 \pm 0.6$ \\
\hline PPL4 & 0 & 15.8 & 0 & 0 \\
\hline
\end{tabular}

$\mathrm{GFP}^{+}$fluorescence intensity (mean \pm SEM) in different subsets of dopaminergic neurons.

the PPM3 neurons is quite low (Fig. 6A,B; two-way ANOVA, $\left.F_{(1,70)}=10.85, p<0.005\right)$. However, at ZT14 when the levels of PDF are low (Park et al., 2000), amount of $\mathrm{GFP}^{+}$signal seen in PPM3 neurons is significantly higher than that at ZT4 (Fig. 6A,B). In the absence of functional PDFR, at both ZT4 and ZT14, the $\mathrm{GFP}^{+}$signal is high and not different from each other (Fig. 6A,B). Importantly, at ZT4, the GFP ${ }^{+}$signal is significantly higher in the absence of functional PDFR than in its presence (Fig. 6). This shows that PDF acting on PDFR in the PPM3 dopaminergic neurons decreases $\mathrm{Ca}^{2+}$ levels specifically during the day time.

\section{A}

TH GAL4 > CaLeXA
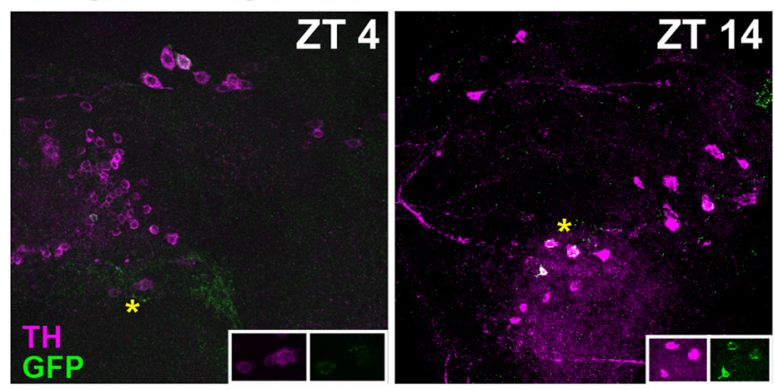

\section{Pdfr5304; TH GAL4 > CaLexA}

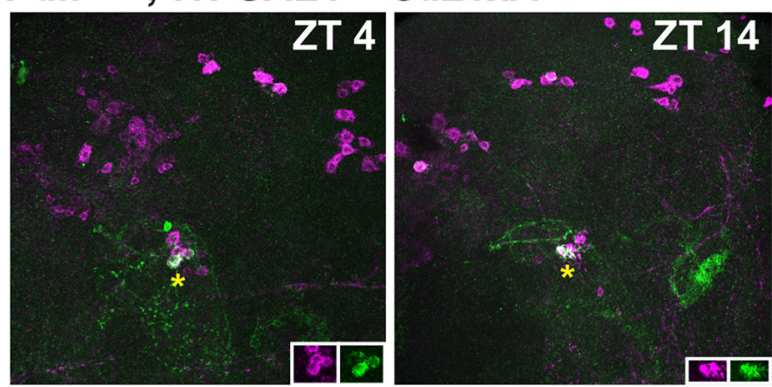

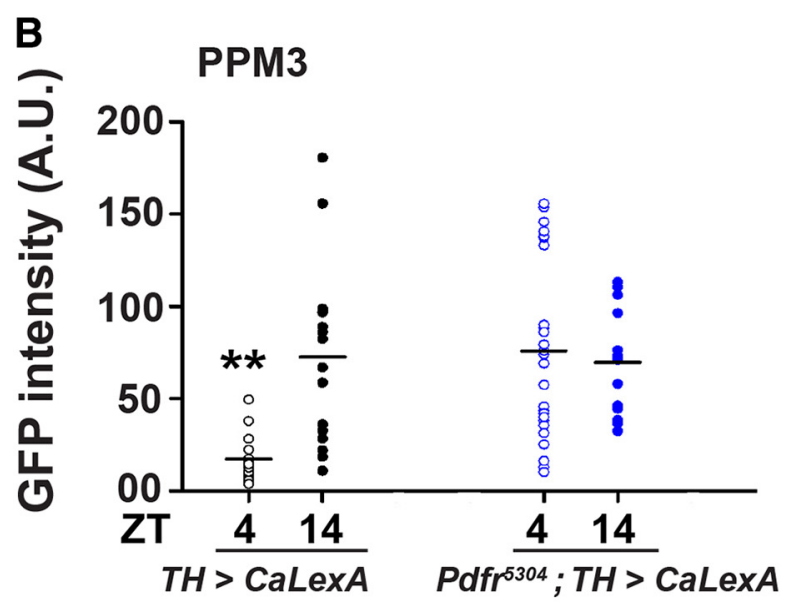

Figure 6. Intracellular $\mathrm{Ca}^{2+}$ levels in PPM3 neurons lower during day than night but remain similar during day and night in the absence of PDFR. A, TH GAL4 expressing CaLexA in WT and pdfr ${ }^{5304}$ backgrounds costained with antibodies against TH to mark dopaminergic neurons and GFP to quantify intracellular $\mathrm{Ca}^{2+}$ levels at two time points: ZT4 and ZT14. CaLexA-driven GFP ${ }^{+}$signal was detected at a low level at ZT4 whereas higher intensity at ZT14 (left panels) in WT background. A.U. = arbitrary units. CaLexA-driven $\mathrm{GFP}^{+}$signal was detected at similar high level at ZT4 and ZT14 (right panels) in pdfr ${ }^{504}$ background. Asterisks indicate PPM3 neurons which are zoomed in inset. $\boldsymbol{B}$, Quantification of results in $\boldsymbol{A}$ shows significantly lower GFP fluorescence in $T H$ GAL4 > CaLexA flies at ZT4 as compared to GFP fluorescence in TH GAL4 > CaLexA flies at ZT14, pdfr ${ }^{5304}$; TH GAL4 > CaLexA flies at ZT4 and ZT14. All other details are as in Figure 1. See also Extended Data Figures 6-1, 6-2. 
Given that dopaminergic neurons are wake-promoting, inhibiting them should inhibit wakefulness. Yet, increasing PDFR in PPM3 neurons which, in accordance with our calcium level quantification should cause increased inhibition, results in decreased day-time sleep. Similarly, absence of pdfr leads to reduced inhibition (as seen from increased $\mathrm{Ca}^{2+}$ levels) of PPM3 neurons, yet behaviourally the flies sleep more during the day time. This indicates that PDF/PDFR signaling is acting on those PPM3 neurons that are, in effect, sleep-promoting. Thus, while a majority of dopaminergic neurons may be wakepromoting there may still be 1-2 PPM3 neurons which are $\mathrm{PDFR}^{+}$and which effectively promote sleep. to examine this heterogeneity, we expressed the Drosophila temperature-sensitive cation channel ITRPA1 (Hamada et al., 2008 ) in different subsets of dopaminergic neurons using $T H-D 1, T H-D$ ' and TH-F3 GAL4 drivers and examined the sleep levels of flies at a low ineffective temperature of $21^{\circ} \mathrm{C}$ as well as at a high activating temperature of $29^{\circ} \mathrm{C}$. As reported in an earlier study (Liu et al., 2012), we find that flies sleep lesser both during the day and night when dopaminergic neurons driven by $T H-D 1$ and TH-D' GAL4 are hyperexcited (Extended Data Fig. 6-2; one-way ANOVA, TH-D1: $F_{(2,76)}=23.16, p<0.00001 ; T H-D^{\prime}: F_{(2,78)}$ $=28.88, p<0.00001)$. However, when neurons expressed by the TH-F3 GAL4 are hyperexcited, flies tend to sleep as much as their GAL4 and UAS parental controls do, especially during the day time (Extended Data Fig. 6 -2; one-way ANOVA, $\left.F_{(2,85)}=2.29, p=0.1\right)$. This can happen only if these neurons do not actually have any effect on sleep, or if they are a heterogeneous group of wake-promoting and sleep-promoting neurons, such that hyperexciting both leads to a cancellation of effects caused by both groups. Given that TH-F3 GAL4-driven dopaminergic neurons have effects on sleep when pdfr levels are altered, our results point toward the possibility of one to two PDFR ${ }^{+}$PPM3 neurons that are also sleeppromoting.

\section{Discussion}

Dopamine is primarily involved in promoting wakefulness (Andretic et al., 2005; Kume et al., 2005) and is known to act on I-LN (Shang et al., 2011) as well as inhibit sleep-promoting dFB (Liu et al., 2012; Ueno et al., 2012) to carry out its wake-promoting function. Here, we uncover that certain dopamine neurons are in fact sleeppromoting and through the inhibitory action of PDFR signaling, wakefulness gets promoted specifically during the day. additional experiments that use optogenetic techniques can shed more light on whether these dopaminergic neurons promote sleep directly, or indirectly by preventing wakefulness either through a gating mechanism or by a permissive role. Interestingly, a previous study has found that dopamine acts on I-LN $\mathrm{v}$ to promote wakefulness (Shang et al., 2011) and we find that PDFR signaling acts on dopamine neurons, suggesting a feedforward pathway for wake promotion, where dopamine acting on I-LN $\mathrm{v}_{\mathrm{v}}$ promotes the inhibition of sleeppromoting dopaminergic neurons by PDFR signaling. The identity of dopamine neurons acting on I-LN $\mathrm{v}$ and those responding to PDFR signaling may differ which can be uncovered with additional experiments.

The role of $s-L N_{v}$ in modulating sleep and wake has been explored in some detail in the recent years. s- $L N_{v}$ have also been shown to promote sleep via short NPF (sNPF) as well as myoinhibitory peptide (MiP) by inhibiting the wake-promoting I-LN $\mathrm{v}_{\mathrm{v}}$ (Shang et al., 2013; Oh et al., 2014). Here, we show that $\mathrm{PDF}^{+} \mathrm{s}-\mathrm{LN}_{\mathrm{v}}$ make synaptic contacts with dopaminergic neurons (Fig. 5) and that PDFR signaling inhibits the downstream dopaminergic neurons (Fig. 6) to promote wakefulness during the day. Moreover, we have shown a secondary role for $s-L N_{v}$ in modulating wake-promoting effects of $\mathrm{I}-\mathrm{LN}_{\mathrm{v}}$. Yet, how this wake-promoting signal which originates in the I-LN $\mathrm{N}_{\mathrm{v}}$ gets relayed to the $s-L_{v}$ is not understood. Furthermore, from our screen it is clear that this function is not mediated via PDFR signaling among the $\mathrm{LN}_{\mathrm{v}}$, as downregulating and overexpressing pdfr in s-LN ${ }_{\mathrm{v}}$ (Clk 9M GAL4 and Pdf GAL4) do not result in any sleep defects. Thus, I-LN $\mathrm{N}_{\mathrm{v}}$ to $s-L N_{v}$ wake-promoting signal is independent of PDF while $s-L_{v}$ to dopamine wake-promoting signal requires PDFR signaling.

PDFR being a class B1 GPCR utilizes cAMP as its second messenger (Shafer et al., 2008; Kunst et al., 2015), although there is evidence for $\mathrm{Ca}^{2+}$ also acting as the second messenger (Mertens et al., 2005). For most of the functions of PDF including stabilizing core clock proteins such as TIMELESS and PERIOD in different target neurons such as $\mathrm{DN}_{1} \mathrm{~s}$ and $\mathrm{s}-\mathrm{LN}_{\mathrm{v}}, \mathrm{CAMP}$ is the major secondary messenger (Li et al., 2014; Seluzicki et al., 2014). Moreover, it is thought that different actions of PDF of slowing and speeding up of morning and evening clock neurons is also mediated by different components of cAMP signaling mechanism (Duvall and Taghert, 2012; Duvall and Taghert, 2013). However, here we show that for the function of regulating wake levels during the day time, PDFR signaling changes levels of intracellular $\mathrm{Ca}^{2+}$ in dopamine neurons with negligible role for CAMP signaling, suggesting a mechanism by which a neuropeptide that has diverse effects on its downstream targets can modulate different functions independently. We therefore identify a unique subset of downstream targets for PDFR signaling among the dopamine neurons that promote wakefulness depending on time of day.

Interestingly, in our screen we note that there are several driver lines using which there are significant changes in day-time sleep but with only one type of manipulation of pdfr levels (Clk 4.1M, 30y, 104y, 121y GAL4). This may be due to ineffective downregulation of pdfr achieved through the Pdfr RNAi line with these particular drivers. Given that PDF is a neuropeptide which can have longrange non-synaptic effects (Nässel and Winther, 2010), even misexpressing it (104y and 121y GAL4) in different substrates has resulted in altered day-time sleep levels. Because DH31 can also respond to PDFR (Kunst et al., 2015), it is possible that these effects could be mediated by DH31 binding to misexpressed PDFR. However, we find that this may not be the case as downregulating DH31-receptor in these regions does not cause changes in sleep levels (data not shown). Thus, we can conclude 
that in regions previously not known to express pdfr, misexpression of pdfr can cause sleep level deficits suggesting that PDF can act in regions which are not direct targets yet may lie in the vicinity of $L N_{v}$ projections.

The role of PDF/PDFR signaling is well-known in synchronizing the free-running molecular rhythms in neurons across the circadian network (Peng et al., 2003; Lin et al., 2004; Wu et al., 2008; Yoshii et al., 2009). PDFR signaling in the "evening" neurons $\left(\mathrm{LN}_{d}\right.$ and $\left.5^{\text {th }} \mathrm{s}-\mathrm{L} \mathrm{N}_{\mathrm{v}}\right)$ is important for appropriate phasing of the evening bout of activity in light/dark cycles (Yao and Shafer, 2014; Guo et al., 2016). While the role of PDF as a wake-signal has been known, here we demonstrate that a subset of dopaminergic neurons is downstream of the PDF/PDFR signaling. While the PDFR expression is not conclusive, we show that perhaps one PPM3 neuron per hemisphere may express the PDFR. Additional experiments that more directly test the functional connectivity between dopaminergic neurons and $\mathrm{PDF}^{+}$neurons, as well as responsiveness of dopaminergic neurons to PDF may result in a clearer picture. Downregulating pdfr in these neurons results in increase of day-time sleep, which is a phenocopy of the sleep behavior of loss-of-function pdfr whole-body mutants. On the other hand, overexpressing pdfr in these neurons leads to decrease of day-time sleep specifically. We further show that PDF and dopaminergic neurons make synaptic contacts with each other at the site of the axonal projection of $s-\mathrm{LN}_{\mathrm{v}}$. Moreover, the effect of PDFR signaling on the PPM3 neurons appears to be inhibitory, suggesting that the PDFR ${ }^{+}$PPM3 neurons promote sleep. Taken together, we conclude that wake-promoting $L_{\mathrm{v}}$ make synaptic connections with sleep-promoting dopaminergic neurons and promote wakefulness specifically during the day time through inhibitory PDFR signaling.

\section{References}

Andretic R, van Swinderen B, Greenspan RJ (2005) Dopaminergic modulation of arousal in Drosophila. Curr Biol 15:1165-1175. CrossRef Medline

Aso Y, Grübel K, Busch S, Friedrich AB, Siwanowicz I, Tanimoto H (2009) The mushroom body of adult Drosophila characterized by GAL4 drivers. J Neurogenet 23:156-172. CrossRef Medline

Bushey D, Huber R, Tononi G, Cirelli C (2007) Drosophila Hyperkinetic mutants have reduced sleep and impaired memory. J Neurosci 27:5384-5393. CrossRef Medline

Cavanaugh DJ, Geratowski JD, Wooltorton JR, Spaethling JM, Hector CE, Zheng X, Johnson EC, Eberwine JH, Sehgal A (2014) Identification of a circadian output circuit for rest:activity rhythms in Drosophila. Cell 157:689-701. CrossRef Medline

Cavanaugh DJ, Vigderman AS, Dean T, Garbe DS, Sehgal A (2016) The Drosophila circadian clock gates sleep through time-of-day dependent modulation of sleep-promoting neurons. Sleep 39:345356. CrossRef Medline

Chung BY, Kilman VL, Keath JR, Pitman JL, Allada R (2009) The GABA(A) receptor RDL acts in peptidergic PDF neurons to promote sleep in Drosophila. Curr Biol 19:386-390. CrossRef Medline

Cirelli C, Bushey D, Hill S, Huber R, Kreber R, Ganetzky B, Tononi G (2005) Reduced sleep in Drosophila shaker mutants. Nature 434: 1087-1092. CrossRef Medline

Crocker A, Sehgal A (2008) Octopamine regulates sleep in Drosophila through protein kinase A-dependent mechanisms. J Neurosci 28:9377-9385. CrossRef Medline
Crocker A, Shahidullah M, Levitan IB, Sehgal A (2010) Identification of a neural circuit that underlies the effects of octopamine on sleep:wake behavior. Neuron 65:670-681. CrossRef Medline

Donlea JM, Thimgan MS, Suzuki Y, Gottschalk L, Shaw PJ (2011) Inducing sleep by remote control facilitates memory consolidation in Drosophila. Science 332:1571-1576. CrossRef Medline

Donlea JM, Pimentel D, Miesenböck G (2014) Neuronal machinery of sleep homeostasis in Drosophila. Neuron 81:1442. CrossRef Medline

Duvall LB, Taghert PH (2012) The circadian neuropeptide PDF signals preferentially through a specific adenylate cyclase isoform AC3 in M pacemakers of Drosophila. PLoS Biol 10:e1001337. CrossRef

Duvall LB, Taghert PH (2013) E and M circadian pacemaker neurons use different PDF receptor signalosome components in Drosophila. J Biol Rhythms 28:239-248. CrossRef

Foltenyi K, Greenspan RJ, Newport JW (2007) Activation of EGFR and ERK by rhomboid signaling regulates the consolidation and maintenance of sleep in Drosophila. Nat Neurosci 10:1160-1167. CrossRef Medline

Gilestro GF, Cirelli C (2009) pySolo: a complete suite for sleep analysis in Drosophila. Bioinformatics 25:1466-1467. CrossRef Medline

Gordon MD, Scott K (2009) Motor control in a Drosophila taste circuit. Neuron 61:373-384. CrossRef Medline

Guo F, Yu J, Jung HJ, Abruzzi KC, Luo W, Griffith LC, Rosbash M (2016) Circadian neuron feedback controls the Drosophila sleepactivity profile. Nature 536:292-297. CrossRef Medline

Hamada FN, Rosenzweig M, Kang K, Pulver SR, Ghezzi A, Jegla TJ, Garrity PA (2008) An internal thermal sensor controlling temperature preference in Drosophila. Nature 454:217-220. CrossRef

He C, Yang Y, Zhang M, Price JL, Zhao Z (2013) Regulation of sleep by neuropeptide Y-like system in Drosophila melanogaster. PLoS One 8:e74237. CrossRef Medline

Hendricks JC, Finn SM, Panckeri KA, Chavkin J, Williams JA, Sehgal A, Pack Al (2000) Rest in Drosophila is a sleep-like state. Neuron 25:129-138. Medline

Hyun S, Lee Y, Hong ST, Bang S, Paik D, Kang J, Shin J, Lee J, Jeon K, Hwang S, Bae E, Kim J (2005) Drosophila GPCR Han is a receptor for the circadian clock neuropeptide PDF. Neuron 48: 267-278. CrossRef Medline

Im SH, Taghert PH (2010) PDF receptor expression reveals direct interactions between circadian oscillators in Drosophila. J Comp Neur 518:1925-1945. CrossRef Medline

Joiner WJ, Crocker A, White BH, Sehgal A (2006) Sleep in Drosophila is regulated by adult mushroom bodies. Nature 441:757-760. CrossRef Medline

Klarsfeld A, Malpel S, Michard-Vanhée C, Picot M, Chélot E, Rouyer $F$ (2004) Novel features of cryptochrome-mediated photoreception in the brain circadian clock of Drosophila. J Neurosci 24:14681477. CrossRef Medline

Kumar S, Chen D, Sehgal A (2012) Dopamine acts through Cryptochrome to promote acute arousal in Drosophila. Genes Dev 26: 1224-1234. CrossRef Medline

Kume K, Kume S, Park SK, Hirsh J, Jackson FR (2005) Dopamine is a regulator of arousal in the fruit fly. J Neurosci 25:7377-7384. CrossRef Medline

Kunst M, Hughes ME, Raccuglia D, Felix M, Li M, Barnett G, Duah J, Nitabach MN (2014) Calcitonin gene-related peptide neurons mediate sleep-specific circadian output in Drosophila. Curr Biol 24: 2652-2664. CrossRef Medline

Kunst M, Tso MC, Ghosh DD, Herzog ED, Nitabach MN (2015) Rhythmic control of activity and sleep by class B1 GPCRs. Crit Rev Biochem Mol Biol 50:18-30. CrossRef Medline

Lear BC, Merrill CE, Lin JM, Schroeder A, Zhang L, Allada R (2005) A G protein-coupled receptor, groom-of-PDF, is required for PDF neuron action in circadian behavior. Neuron 48:221-227. CrossRef

Li Y, Guo F, Shen J, Rosbash M (2014) PDF and cAMP enhance PER stability in Drosophila clock neurons. Proc Natl Acad Sci USA 111:E1284-E1290. CrossRef Medline 
Lin Y, Stormo GD, Taghert PH (2004) The neuropeptide pigmentdispersing factor coordinates pacemaker interactions in the Drosophila circadian system. J Neurosci 24:7951-7957. CrossRef Medline

Liu Q, Liu S, Kodama L, Driscoll MR, Wu MN (2012) Two dopaminergic neurons signal to the dorsal fan-shaped body to promote wakefulness in Drosophila. Curr Biol 22:2114-2123. CrossRef Medline

Liu S, Lamaze A, Liu Q, Tabuchi M, Yang Y, Fowler M, Bharadwaj R, Zhang J, Bedont J, Blackshaw S, Lloyd TE, Montell C, Sehgal A, Koh K, Wu MN (2014) WIDE AWAKE mediates the circadian timing of sleep onset. Neuron 82:151-166. CrossRef Medline

Liu S, Liu Q, Tabuchi M, Wu MN (2016) Sleep drive is encoded by neural plastic changes in a dedicated circuit. Cell 165:1347-1360. CrossRef Medline

Mao Z, Davis RL (2009) Eight different types of dopaminergic neurons innervate the Drosophila mushroom body neuropil: anatomical and physiological heterogeneity. Front Neural Circuits 3:5. CrossRef Medline

Masuyama K, Zhang Y, Rao Y, Wang JW (2012) Mapping neural circuits with activity-dependent nuclear import of a transcription factor. J Neurogenet 26:89-102. CrossRef

Mertens I, Vandingenen A, Johnson EC, Shafer OT, Li W, Trigg JS, De Loof A, Schoofs L, Taghert PH (2005) PDF receptor signaling in Drosophila contributes to both circadian and geotactic behaviors. Neuron 48:213-219. CrossRef Medline

Nässel DR, Winther AM (2010) Drosophila neuropeptides in regulation of physiology and behavior. Prog Neurobiol 92:42-104. CrossRef Medline

Nitabach MN, Blau J, Holmes TC (2002) Electrical silencing of Drosophila pacemaker neurons stops the free-running circadian clock. Cell 109:485-495.

Nitabach MN, Wu Y, Sheeba V, Lemon WC, Strumbos J, Zelensky PK, White BH, Holmes TC (2006) Electrical hyperexcitation of lateral ventral pacemaker neurons desynchronizes downstream circadian oscillators in the fly circadian circuit and induces multiple behavioral periods. J Neurosci 26:479-489.

Oh Y, Yoon SE, Zhang Q, Chae HS, Daubnerová I, Shafer OT, Choe J, Kim YJ (2014) A homeostatic sleep-stabilizing pathway in Drosophila composed of the sex peptide receptor and its ligand, the myoinhibitory peptide. PLoS Biol 12:e1001974. CrossRef

Parisky KM, Agosto J, Pulver SR, Shang Y, Kuklin E, Hodge JJ, Kang K, Liu X, Garrity PA, Rosbash M, Griffith LC (2008) PDF cells are a GABA-responsive wake-promoting component of the Drosophila sleep circuit. Neuron 60:672-682. CrossRef Medline

Park JH, Helfrich-Förster C, Lee G, Liu L, Rosbash M, Hall JC (2000) Differential regulation of circadian pacemaker output by separate clock genes in Drosophila. Proc Natl Acad Sci USA 97:3608-3613. CrossRef Medline

Peng Y, Stoleru D, Levine JD, Hall JC, Rosbash M (2003) Drosophila free-running rhythms require intercellular communication. PLoS Biol 1:E13. CrossRef Medline

Pitman JL, McGill JJ, Keegan KP, Allada R (2006) A dynamic role for the mushroom bodies in promoting sleep in Drosophila. Nature 441:753-756. CrossRef Medline
Potdar S, Sheeba V (2012) Large ventral lateral neurons determine the phase of evening activity peak across photoperiods in Drosophila melanogaster. J Biol Rhythms 27:267-279. CrossRef Medline

Seluzicki A, Flourakis M, Kula-Eversole E, Zhang L, Kilman V, Allada R (2014) Dual PDF signaling pathways reset clocks via TIMELESS and acutely excite target neurons to control circadian behavior. PLoS Biol 12:e1001810. CrossRef Medline

Shafer OT, Kim DJ, Dunbar-Yaffe R, Nikolaev VO, Lohse MJ, Taghert PH (2008) Widespread receptivity to neuropeptide PDF throughout the neuronal circadian clock network of Drosophila revealed by real-time cyclic AMP imaging. Neuron 58:223-237. CrossRef Medline

Shang Y, Griffith LC, Rosbash M (2008) Light-arousal and circadian photoreception circuits intersect at the large PDF cells of the Drosophila brain. Proc Natl Acad Sci USA 105:19587-19594. CrossRef Medline

Shang Y, Haynes P, Pírez N, Harrington Kl, Guo F, Pollack J, Hong P, Griffith LC, Rosbash M (2011) Imaging analysis of clock neurons reveals light buffers the wake-promoting effect of dopamine. Nat Neurosci 14:889-895. CrossRef Medline

Shang Y, Donelson NC, Vecsey CG, Guo F, Rosbash M, Griffith LC (2013) Short neuropeptide $F$ is a sleep-promoting inhibitory modulator. Neuron 80:171-183. CrossRef Medline

Shaw PJ, Cirelli C, Greenspan RJ, Tononi G (2000) Correlates of sleep and waking in Drosophila melanogaster. Science 287:18341837. Medline

Sheeba V, Fogle KJ, Kaneko M, Rashid S, Chou YT, Sharma VK, Holmes TC (2008) Large ventral lateral neurons modulate arousal and sleep in Drosophila. Curr Biol 18:1537-1545. CrossRef Medline

Sheeba V, Fogle KJ, Holmes TC (2010) Persistence of morning anticipation behavior and high amplitude morning startle response following functional loss of small ventral lateral neurons in Drosophila. PLoS One 5:e11628. CrossRef Medline

Ueno T, Tomita J, Tanimoto H, Endo K, Ito K, Kume S, Kume K (2012) Identification of a dopamine pathway that regulates sleep and arousal in Drosophila. Nat Neurosci 15:1516-1523. CrossRef Medline

Wu Y, Cao G, Nitabach MN (2008) Electrical silencing of PDF neurons advances the phase of non-PDF clock neurons in Drosophila. J Biol Rhythms 23:117-128. CrossRef Medline

Yao Z, Shafer OT (2014) The Drosophila circadian clock is a variably coupled network of multiple peptidergic units. Science 343:15161520. CrossRef Medline

Yoshii T, Wulbeck C, Sehadova H, Veleri S, Bichler D, Stanewsky R, Helfrich-Forster C (2009) The neuropeptide pigment-dispersing factor adjusts period and phase of Drosophila's clock. J Neurosci 29:2597-2610. CrossRef

Yuan Q, Joiner WJ, Sehgal A (2006) A sleep-promoting role for the Drosophila serotonin receptor 1A. Curr Biol 16:1051-1062. CrossRef Medline

Zhang L, Chung BY, Lear BC, Kilman VL, Liu Y, Mahesh G, Meissner RA, Hardin PE, Allada R (2010) DN1(p) circadian neurons coordinate acute light and PDF inputs to produce robust daily behavior in Drosophila. Curr Biol 20:591-599. CrossRef Medline 